\title{
Nonspecial varieties and generalised Lang-Vojta conjectures
}

\author{
Erwan Rousseau $^{1,2}$, Amos Turchet ${ }^{(\mathbb{D})}$ and Julie Tzu-Yueh Wang ${ }^{4}$ \\ ${ }^{1}$ Institut Universitaire de France \& Aix-Marseille Université, CNRS, Centrale Marseille, I2M, Marseille, France; \\ E-mail: erwan.rousseau@univ-amu.fr. \\ ${ }^{2}$ Freiburg Institute for Advanced Studies, University of Freiburg, Albertstr. 19,79104 Freiburg, Germany. \\ ${ }^{3}$ Dipartimento di Matematica e Fisica, Universitá degli studi Roma 3, L.go S. L. Murialdo 1,00146 Roma, Italy; \\ E-mail: amos.turchet@uniroma3.it. \\ ${ }^{4}$ Institute of Mathematics, Academia Sinica No. 1, Sec. 4, Roosevelt Road Taipei 10617, Taiwan; \\ E-mail: jwang@math.sinica.edu.tw.
}

Received: 28 August 2020; Accepted: 25 December 2020

2020 Mathematics Subject Classification: Primary - 14G40; Secondary - 11J97, 14G05, 32A22

Keywords: Campana's conjectures; Function fields; Nevanlinna Theory; Orbifolds; Hyperbolicity

\begin{abstract}
We construct a family of fibred threefolds $X_{m} \rightarrow(S, \Delta)$ such that $X_{m}$ has no étale cover that dominates a variety of general type but it dominates the orbifold $(S, \Delta)$ of general type. Following Campana, the threefolds $X_{m}$ are called weakly special but not special. The Weak Specialness Conjecture predicts that a weakly special variety defined over a number field has a potentially dense set of rational points. We prove that if $m$ is big enough, the threefolds $X_{m}$ present behaviours that contradict the function field and analytic analogue of the Weak Specialness Conjecture. We prove our results by adapting the recent method of Ru and Vojta. We also formulate some generalisations of known conjectures on exceptional loci that fit into Campana's program and prove some cases over function fields.
\end{abstract}

\section{Introduction}

A fundamental problem in Diophantine geometry is to describe the distribution of rational points in an algebraic variety $X$ defined over a number field $k$. The expectation is that the global geometry of $X$ controls the distribution of $X(k)$. In the case in which $X$ is a variety of general type, conjectures of Lang and Vojta (see, for example, [Lan91, Conjecture 3.7], [Voj87, Conjecture 3.4.3]) predict the existence of a proper Zariski closed subset, called the exceptional set, such that in its complement rational points $X(L)$ should be finite for every finite extension $L \supset k$; moreover, the exceptional set is expected to be independent of the field of definition. On the opposite side, there are the varieties whose rational points are potentially dense - that is, there exists a finite field extension $L / k$ such that the set of $L$-rational points $X(L)$ is Zariski dense. The conjectures of Lang and Vojta thus predict that rational points in a variety of general type are not potentially dense.

It is natural to look for geometrical properties characterising varieties where the set of rational points is potentially dense. The conjectures mentioned imply that for such a property to be satisfied on $X$, not only $X$ but none of its finite étale covers should dominate a positive-dimensional variety of general type: such varieties are called weakly special (see Section 4 for details). In [HT00, Conjecture 1.2], the following conjecture was stated, which we will call the Weak Specialness Conjecture: a variety $X$ has a potentially dense set of rational points if and only if $X$ is weakly special.

(C) The Author(s), 2021. Published by Cambridge University Press. This is an Open Access article, distributed under the terms of the Creative Commons Attribution licence (http://creativecommons.org/licenses/by/4.0/), which permits unrestricted re-use, distribution, and reproduction in any medium, provided the original work is properly cited. 
In [Cam04], Campana introduced a stronger notion of specialness: a variety $X$ is special if it does not admit any fibration of general type, in the sense of Campana (see Section 2 for details). Campana conjectured that specialness, rather than weak specialness, should characterise potential density. Note that these two characterisations differ from each other (even if they agree in dimension up to 2), because there exist projective varieties which are weakly special but not special. Such examples were first constructed in [BT04] as simply connected threefolds equipped with an elliptic fibration $\pi: X \rightarrow S$, where $S$ has Kodaira dimension 1 . Nevertheless, these examples do not explicitly contradict the Weak Specialness Conjecture, since we still lack a method to control the distribution of rational points on these varieties. Therefore it is natural to consider the analogous problem in the analytic and functionfield settings. The goal of this article is to study the two conjectures in these settings, giving evidence for Campana's.

In the analytic setting, the Green-Griffiths-Lang conjecture predicts that entire curves in varieties of general type should be contained in a proper Zariski closed subset, the already mentioned exceptional set. Following this analogy, Campana has conjectured that specialness (and therefore potential density) should correspond to the existence of Zariski dense entire curves (see [Cam04]). The analytic analogue of the Weak Specialness Conjecture would imply that a weakly special variety admits a Zariski dense entire curve. This was already disproved in [CP07], where it was proven that for some of the examples of weakly special but not special varieties constructed in [BT04], all entire curves are algebraically degenerate (that is, with non-Zariski dense image).

Similarly, the conjectures of Lang and Vojta admit analogues for varieties defined over function fields. In our setting, Vojta's height conjecture predicts that given a general type variety $X$ defined over a characteristic zero function field $\kappa(\mathcal{C})$ of a smooth integral curve $\mathcal{C}$, and an ample line bundle $\mathcal{L}$, there exists a positive constant $\alpha$ such that sections $s: \mathcal{C} \rightarrow X$ not contained in the exceptional set satisfy $\operatorname{deg} s^{*} \mathcal{L} \leq \alpha(2 g(\mathcal{C})-2)$. We say that varieties $X$ satisfying this condition are pseudo-algebraically hyperbolic and we refer to Definition 2.9 for further details and references. A function-field analogue of the Weak Specialness Conjecture would predict that a weakly special variety $X$ does not dominate a positive-dimensional pseudo-algebraically hyperbolic variety.

One of the goals of this paper is to present a list of new examples of varieties that are weakly special but not special: these are of the form $\pi: X_{m} \rightarrow\left(S, \Delta_{\pi}\right)$, where $X_{m}$ is a threefold, $\pi$ is an elliptic fibration, the compactification $\bar{S}$ of $S$ is a blowup of $\mathbb{P}^{2}$ and $\Delta_{\pi}=(1-1 / m) \widetilde{D_{1}}$ is an orbifold divisor (see Section 6 for the precise definition).

The pairs $\left(S, \Delta_{\pi}\right)$ can be seen as orbifold generalisations of surfaces appearing in [CZ10], which provides examples of simply connected quasi-projective surfaces with a non-Zariski dense set of integral points. For this class of examples we show that the function-field and analytic Weak Specialness Conjectures fail. In particular, we prove the following:

Theorem 1.1 (see Proposition 6.2 and Theorem 6.5). In the settings already given, there exists $m_{0}$ such that for all $m \geq m_{0}$, the following hold:

1. the base of the fibration $X_{m} \rightarrow\left(S, \Delta_{\pi}\right)$ is pseudo-algebrically hyperbolic and

2. every entire curve in $X_{m}$ is algebraically degenerate.

As an example we obtain the following explicit corollary:

Corollary 1.2. Let $L_{1}, L_{2}, L_{3}$ and $L_{4}$ be four lines in general position in $\mathbb{P}^{2}$ and let $\bar{S}$ be the blowup of $\mathbb{P}^{2}$ in three points $P_{1}, P_{2}, P_{3}$, where $P_{i} \in L_{i}$ for $i=1,2,3$. If we denote by $\tilde{L}_{i}$ the strict transform of the line $L_{i}$, and $S=\bar{S} \backslash \tilde{L}_{2}+\tilde{L}_{3}+\tilde{L}_{4}$, the surface $S \backslash \tilde{L}_{1}$ is simply connected and the orbifold $\left(\bar{S}, \Delta_{m}=(1-1 / m) \tilde{L}_{1}+\tilde{L}_{2}+\tilde{L}_{3}+\tilde{L}_{4}\right)$ is of general type for every $m \geq 2$-that is, the divisor $K_{\bar{S}}+\Delta_{m}$ is big. Then $X_{m}=\left(T \times_{\mathbb{P}_{1}} \bar{S}\right) \rightarrow\left(\bar{S}, \Delta_{m}\right)$, where $T$ is an elliptic fibration (see Example 4.3 for the construction), is a simply connected threefold that does not dominate any surface or curve of general type, and therefore it is weakly special (see Theorem 4.2). Then the following hold:

1. a degree bound for maps of the form $\pi \circ s: \mathcal{C} \rightarrow\left(\bar{S}, \Delta_{m}\right)$ and

2. algebraic degeneracy of entire curves in $X_{m}$. 
In [HT01, Problem 3.7], Hassett and Tschinkel ask whether a pair that does not admit any étale cover that dominates a variety of log general type - that is, a weakly special one - admits a potentially dense set of integral points. Theorem 1.1 answers the analogous question over function fields in the negative.

To prove Theorem 1.1 we combine Corvaja and Zannier's degeneracy statements in [CZ04] with the recent framework of Ru and Vojta [RV20] to obtain a generalisation of [CZ04, Main Theorem] in the orbifold setting for the function-field and analytic cases.

Theorem 1.3 (see Corollary 5.8 and Theorem 5.11). Let $X \subset \mathbb{P}^{m}$ be a complex nonsingular projective surface and $D=D_{1}+\cdots+D_{q}$ be a divisor with $q \geq 2$, such that

1. no three of the components $D_{i}$ meet at a point and

2. there exists a choice of positive integers $p_{i}$ such that

$\circ$ the divisor $D_{p}:=p_{1} D_{1}+p_{2} D_{2}+\cdots+p_{q} D_{q}$ is ample and

$\circ$ the inequality

$$
2 D_{p}^{2} \xi_{i}>\left(D_{p} \cdot D_{i}\right) \xi_{i}^{2}+3 D_{p}^{2} p_{i}
$$

holds for every $i=1, \ldots, q$, where $\xi_{i}$ is the minimal positive solution of the equation $D_{i}^{2} x^{2}-2\left(D_{p} \cdot D_{i}\right) x+D_{p}^{2}=0$.

Let $\Delta$ be the $\mathbb{Q}$-divisor defined as

$$
\Delta=\sum_{i=1}^{q}\left(1-\frac{1}{m_{i}}\right) D_{i} .
$$

Then there exists a positive integer $m$ such that if $m_{i} \geq m$ for every $i$,

1. every orbifold entire curve $\psi: \mathbb{C} \rightarrow(X, \Delta)$, is algebraically degenerate and

2. $(X, \Delta)$ is pseudo-algebraically hyperbolic.

The key point in the proof of Theorem 1.3 is an analytic and a function-field version of the $\mathrm{Ru}-$ Vojta's Theorem [RV20, General Theorem] with truncation, which we develop in Theorems 7.1 and 7.6 by adding a ramification term to the $\mathrm{Ru}$-Vojta Theorem. Even though this type of generalisation is far from reach in the arithmetic setting, our approach via the Ru-Vojta Theorem gives a new interpretation of some important work, such as [CZ04], that has contributed fundamental ideas and techniques in the development of the Ru-Vojta Theorem. We expect that this point of view and the truncated version of the $\mathrm{Ru}-$ Vojta Theorem will have further applications.

\section{Structure of the paper}

In Section 2 we recall basic facts about special varieties and orbifolds, following Campana. Then in Section 3 we present a general framework where we generalise Lang's notion of exceptional loci to nonspecial varieties and Campana orbifolds. In Section 4 we discuss a general procedure to construct weakly special but not special varieties, generalising [BT04]. In Section 5 we prove Theorem 1.3, and we apply it in Section 6 to prove Theorem 1.1. Finally, in Section 7 we prove some generalisations of Ru and Vojta [RV20, General Theorem] for function fields and entire curves.

\section{Special varieties}

We collect here basic definitions and constructions related to special varieties, while referring to [Cam04] for more details.

\subsection{Special manifolds via Bogomolov sheaves}

Let $X$ be a connected complex nonsingular projective variety of complex dimension $n$. For a rank 1 coherent subsheaf $\mathcal{L} \subset \Omega_{X}^{p}$, denote by $H^{0}\left(X, \mathcal{L}^{m}\right)$ the space of sections of $\operatorname{Sym}^{m}\left(\Omega_{X}^{p}\right)$ which take values 
in $\mathcal{L}^{m}$ (where, as usual, $\mathcal{L}^{m}:=\mathcal{L}^{\otimes m}$ ). The Iitaka dimension of $\mathcal{L}$ is $\kappa(X, \mathcal{L}):=\max _{m>0}\left\{\operatorname{dim}\left(\Phi_{\mathcal{L}^{m}}(X)\right)\right\}$ - that is, the maximum dimension of the image of rational maps $\Phi_{\mathcal{L}^{m}}: X \rightarrow \mathbb{P}\left(H^{0}\left(X, \mathcal{L}^{m}\right)\right)$ defined at the generic point of $X$, where by convention $\operatorname{dim}\left(\Phi_{\mathcal{L}^{m}}(X)\right):=-\infty$ if there are no global sections. Thus $\kappa(X, \mathcal{L}) \in\{-\infty, 0,1, \ldots, \operatorname{dim}(X)\}$. In this setting, a theorem of Bogomolov in [Bog79] shows that if $\mathcal{L} \subset \Omega_{X}^{p}$, then $\kappa(X, \mathcal{L}) \leq p$.

Definition 2.1. Let $p>0$. A rank 1 saturated coherent sheaf $\mathcal{L} \subset \Omega_{X}^{p}$ is called a Bogomolov sheaf if $\kappa(X, \mathcal{L})=p$ - that is, if $\mathcal{L}$ has the largest possible Iitaka dimension.

The following remark shows that the presence of Bogomolov sheaves on $X$ is related to the existence of fibrations $f: X \rightarrow Y$, where $Y$ is of general type:

Remark 2.2. If $f: X \rightarrow Y$ is a fibration (by which we mean a surjective morphism with connected fibres) and $Y$ is a variety of general type of dimension $p>0$, then the saturation of $f^{*}\left(K_{Y}\right)$ in $\Omega_{X}^{p}$ is a Bogomolov sheaf of $X$.

Campana introduced the notion of specialness in [Cam04, Definition 2.1] to generalise the absence of fibration.

Definition 2.3. A nonsingular variety $X$ is said to be special (or of special type) if there is no Bogomolov sheaf on $X$. A projective variety is said to be special if some (or any) of its resolutions are special.

By the previous remark, if there is a fibration $X \rightarrow Y$ with $Y$ a positive-dimensional variety of general type, then $X$ is nonspecial. In particular, if $X$ is of general type of positive dimension, $X$ is not special.

\subsection{Special manifolds via orbifold bases}

Campana gave a characterisation of special varieties using his theory of orbifolds. We briefly recall the construction.

Let $Z$ be a normal connected compact complex variety. An orbifold divisor $\Delta$ is a linear combination $\Delta:=\sum_{\{D \subset Z\}} c_{\Delta}(D) \cdot D$, where $D$ ranges over all prime divisors of $Z$ and the orbifold coefficients are rational numbers $c_{\Delta}(D) \in[0,1] \cap \mathbb{Q}$ such that all but finitely many are zero. Equivalently,

$$
\Delta=\sum_{\{D \subset Z\}}\left(1-\frac{1}{m_{\Delta}(D)}\right) \cdot D,
$$

where only finitely many orbifold multiplicities $m_{\Delta}(D) \in \mathbb{Q}_{\geq 1} \cup\{+\infty\}$ are larger than 1 .

An orbifold pair is a pair $(Z, \Delta)$ where $\Delta$ is an orbifold divisor; they interpolate between the compact case on the one hand, where $\Delta=\varnothing$ and the pair $(Z, \varnothing)=Z$ has no orbifold structure, and on the other hand the open, or purely logarithmic case, where $c_{j}=1$ for all $j$, and we identify $(Z, \Delta)$ with $Z \backslash \operatorname{Supp}(\Delta)$.

When $Z$ is smooth and the support $\operatorname{Supp}(\Delta):=\cup D_{j}$ of $\Delta$ has normal crossing singularities, we say that $(Z, \Delta)$ is smooth. When all multiplicities $m_{j}$ are integral or $+\infty$, we say that the orbifold pair $(Z, \Delta)$ is integral, and when every $m_{j}$ is finite, it may be thought of as a virtual ramified cover of $Z$ ramifying at order $m_{j}$ over each of the $D_{j}$ s.

Consider a fibration $f: X \rightarrow Z$ between normal connected complex projective varieties. In general, the geometric invariants of $X$ (such as $\pi_{1}(X), \kappa(X), \ldots$ ) do not coincide with the 'sum' of those of the base $(Z)$ and of the generic fibre $\left(X_{\eta}\right)$ of $f$. Replacing $Z$ by the 'orbifold base' $\left(Z, \Delta_{f}\right)$ of $f$, which encodes the multiple fibres of $f$, leads in some favorable cases to such an additivity (on suitable birational models, at least).

Definition 2.4 (Orbifold base of a fibration). Let $f: X \rightarrow Z$ be a fibration as before and let $\Delta$ be an orbifold divisor on $X$. We shall define the orbifold base $\left(Z, \Delta_{f}\right)$ of $(f, \Delta)$ as follows: to each irreducible Weil divisor $D \subset Z$ we assign the multiplicity $m_{(f, \Delta)}(D):=\inf _{k}\left\{t_{k} \cdot m_{\Delta}\left(F_{k}\right)\right\}$, where the scheme-theoretic fibre of $D$ is $f^{*}(D)=\sum_{k} t_{k} \cdot F_{k}+R, R$ is an $f$-exceptional divisor of $X$ with $f(R) \subsetneq D$ 
and $F_{k}$ are the irreducible divisors of $X$ which map surjectively to $D$ via $f$.

Remark 2.5. Note that the integers $t_{k}$ are well defined, even if $X$ is only assumed to be normal.

Let $(Z, \Delta)$ be an orbifold pair. Assume that $K_{Z}+\Delta$ is $\mathbb{Q}$-Cartier (this is the case if $(Z, \Delta)$ is smooth, for example): we will call it the canonical bundle of $(Z, \Delta)$. Similarly, we will denote by the canonical dimension of $(Z, \Delta)$ the Kodaira dimension of $K_{Z}+\Delta-$ that is, $\kappa(Z, \Delta):=\kappa\left(Z, \mathcal{O}_{Z}\left(K_{Z}+\Delta\right)\right)$. Finally, we say that the orbifold $(Z, \Delta)$ is of general type if $\kappa(Z, \Delta)=\operatorname{dim}(Z)$.

Definition 2.6. A fibration $f: X \rightarrow Z$ is said to be of general type if $Z$ is positive-dimensional and the orbifold base $\left(Z, \Delta_{f}\right)$ is of general type.

Campana's idea was that in order to characterise varieties that have a potentially dense set of rational points, it was not sufficient to exclude the presence of étale covers that dominate varieties of general type. One would need to exclude the presence of every fibration of general type in the sense already discussed. This turns out to be equivalent to the specialness condition of Definition 2.3 as proven by Campana.

Theorem 2.7 (see [Cam04, Theorem 2.27]). A variety $X$ is special if and only if it has no fibrations of general type.

\subsection{Orbifold morphisms}

We recall here the main definition of orbifold morphism, following [Cam11, Definition 2.3], in the special case in which the source is a curve.

Definition 2.8. Let $\left(X, \Delta_{X}\right)$ and $\left(\mathcal{C}, \Delta_{\mathcal{C}}\right)$ be two orbifold pairs, with $X$ and $\mathcal{C}$ normal projective varieties defined over a field $k, \mathcal{C}$ a curve and $\Delta_{X}, \Delta_{\mathcal{C}}$ two orbifold divisors of the form

$$
\Delta_{X}=\sum_{i=1}^{r}\left(1-\frac{1}{m_{i}}\right) \Delta_{i}, \quad \Delta_{\mathcal{C}}=\sum_{j=1}^{s}\left(1-\frac{1}{n_{j}}\right) P_{j} .
$$

For every prime divisor $D$ of $X$ we denote by $m_{X}(D)$ its multiplicity - that is, the number $m_{i}$ if $D=\Delta_{i}$ or 0 otherwise. For every point $Q \in \mathcal{C}$ we similarly define $n_{\mathcal{C}}(Q)$. A morphism $f: \mathcal{C} \rightarrow X$ is an orbifold morphism, denoted by $f:\left(\mathcal{C}, \Delta_{\mathcal{C}}\right) \rightarrow\left(X, \Delta_{X}\right)$, if

1. $f(\mathrm{C})$ is not contained in $\Delta_{X}$ and

2. for every prime divisor $D$ of $X$, if $f^{*} D=\sum_{k} n_{k} Q_{k}$, then we have $n_{k} \cdot n_{\mathcal{C}}\left(Q_{k}\right) \geq m_{X}(D)$.

We recall the following definition introduced by Demailly in [Dem97] in the compact case (the logarithmic and orbifold analogues were introduced in [Che04] and [Rou10], respectively):

Definition 2.9. Let $(X, D)$ be a pair of a nonsingular projective variety $X$ defined over $k$ and let $D$ be a normal crossing divisor on $X$. We say that $(X, D)$ is algebraically hyperbolic if there exists an ample line bundle $\mathcal{L}$ on $X$ and a positive constant $\alpha$ such that for every nonsingular projective curve $\mathcal{C}$ and every morphism $\varphi: \mathcal{C} \rightarrow X$, the following holds:

$$
\operatorname{deg} \varphi^{*} \mathcal{L} \leq \alpha \cdot\left(2 g(\mathcal{C})-2+N_{\varphi}^{[1]}(D)\right)
$$

where $N_{\varphi}^{[1]}(D)$ is the cardinality of the support of $\varphi^{*}(D)$. We say that $(X, D)$ is pseudo-algebraically hyperbolic if there exists a proper closed subvariety $Z$ of $X$ such that formula (2.1) holds for every morphism $\varphi: \mathcal{C} \rightarrow X$ such that $\varphi(\mathcal{C}) \nsubseteq Z$.

The notion of pseudo-algebraic hyperbolicity was defined first in [vBJK19, JX20]. In Lang's terminology [Lan86], the notion is the 'pseudofication' of the notion of algebraic hyperbolicity. We note that when equation (2.1) holds for an ample line bundle $\mathcal{L}$, it holds for every ample line bundle with possibly a different constant $\alpha$. 
Remark 2.10. Note that the degree $\operatorname{deg} \varphi^{*} \mathcal{L}$ is a Weil height for $\varphi$ viewed as a point in the function field $k(\mathcal{C})$, with respect to the ample line bundle $\mathcal{L}$. In the next sections, we refer to it both as a degree and as the height $h_{\mathcal{L}}(\varphi)$.

Definition 2.9 can be extended to the orbifold setting as follows:

Definition 2.11. Let $(X, \Delta)$ be an orbifold; we say that $(X, \Delta)$ is algebraically hyperbolic if there exists an ample line bundle $\mathcal{L}$ on $X$ and a positive constant $\alpha$ such that for every nonsingular projective curve $\mathcal{C}$ and every orbifold morphism $\psi:\left(\mathcal{C}, \Delta_{C}\right) \rightarrow(X, \Delta)$, the following holds:

$$
\operatorname{deg} \psi^{*} \mathcal{L} \leq \alpha \cdot\left(2 g(\mathcal{C})-2+\operatorname{deg} \Delta_{C}\right) .
$$

We say that $(X, \Delta)$ is pseudo-algebraically hyperbolic if there exists a proper closed subvariety $Z$ of $X$ such that formula (2.2) holds for every orbifold morphism $\psi:\left(\mathcal{C}, \Delta_{C}\right) \rightarrow(X, \Delta)$ such that $\psi(\mathcal{C}) \nsubseteq Z$.

\section{Generalised Lang conjectures}

In this section we propose a generalisation of the conjectures of Lang and Vojta compatible with Campana's dichotomy between special and nonspecial.

\subsection{Arithmetic and analytic exceptional sets}

In [Lan86], Lang introduced the following exceptional sets:

Definition 3.1. Let $X$ be a projective variety defined over a field $k$.

1. If $k$ is a finitely generated field of characteristic 0 , then the Diophantine exceptional set $\operatorname{Exc}_{\text {dio }}(X)$ is the smallest Zariski closed subset $Z$ of $X$ such that $(X \backslash Z)(L)$ is finite for all finite extensions $L \supset k$.

2. If $k=\mathbb{C}$, then the holomorphic exceptional set $\operatorname{Exc}_{\text {hol }}(X)$ is the Zariski closure of the union of all entire curves - that is, images of nonconstant holomorphic maps $f: \mathbb{C} \rightarrow X$.

Lang conjectured [Lan86] that $X$ is of general type if and only if both these exceptional sets are proper subsets of $X$. Moreover, he conjectured that $\operatorname{Exc}_{\text {dio }}(X)=\operatorname{Exc}_{\text {hol }}(X)$.

Given Campana's notion of special variety, it is natural to try to extend the notion of exceptional sets to nonspecial varieties in the sense of Campana [Cam04].

The starting point is the following conjecture, formulated by Campana [Cam04, Conjecture 9.2 and Conjecture 9.20]:

Conjecture 1. Let $X$ be a projective variety defined over a field $k$.

1. If $k$ is a number field, then $X(L)$ is not Zariski dense for all finite extensions $L \supset k$ if and only if $X$ is not special.

2. If $k=\mathbb{C}$, then $f(\mathbb{C})$ is not Zariski dense for all entire curves $f: \mathbb{C} \rightarrow X$ if and only if $X$ is not special.

When one considers nonspecial varieties, it is easy to see that there are examples of nonspecial varieties where the two exceptional sets in Definition 3.1 are the entire variety. As an example, consider the nonspecial variety $X=\mathcal{C} \times \mathbb{P}^{1}$ defined over a number field $k$, where $\mathcal{C}$ is a smooth projective curve of genus $\geq 2$ : in this case, for every rational point $P$ in $\mathcal{C}(L)$, the curve $\{P\} \times \mathbb{P}^{1}$ is in $\operatorname{Exc}_{\text {dio }}(X)$. Therefore, since $X(\bar{k})$ is dense in $X$, Lang's Diophantine exceptional set coincides with $X$. Similarly, one can show that $\operatorname{Exc}_{\text {hol }}(X)=X$. We suggest that in order to define meaningful exceptional sets, one should consider the projectivised tangent bundle $\mathbb{P}\left(T_{X}\right)$.

\section{Definition 3.2.}

1. If $k$ is a number field, the Diophantine exceptional $\operatorname{set}^{\operatorname{Exc}_{\text {dio }}^{1}}(X)$ is the smallest Zariski closed subset $Z$ of $\mathbb{P}\left(T_{X}\right)$ such that for all finite extensions $L \supset k, \mathbb{P}\left(T_{Y_{L}}\right) \subset Z$, where $Y_{L}$ is the Zariski closure of $X(L)$. 
2. If $k=\mathbb{C}$, the holomorphic exceptional set $\operatorname{Exc}_{\text {hol }}^{1}(X)$ is the Zariski closure of the union of all entire curves $g: \mathbb{C} \rightarrow \mathbb{P}\left(T_{X}\right)$ obtained as liftings of entire curves $f: \mathbb{C} \rightarrow X$.

The main motivation behind Definition 3.2 is Campana's core construction in [Cam04, Section 3]. Given a smooth projective variety $X$, there is a functorial fibration $c_{X}: X \rightarrow C(X)$, called the core of $X$, such that the fibres of $c_{X}$ are special varieties and the base $C(X)$ is either a point or an orbifold of general type. The idea behind considering $\mathbb{P}\left(T_{X}\right)$ as the natural space where the exceptional set lives is that the core of $X$ identifies the 'special direction' in $\mathbb{P}\left(T_{X}\right)$ and therefore, assuming that $C(X)$ has positive dimension - that is, that $X$ is nonspecial - this exceptional set should not be the whole $\mathbb{P}\left(T_{X}\right)$.

Therefore we propose the following generalisation of Lang's conjecture for nonspecial varieties:

Conjecture 2. Let $X$ be a projective variety defined over a field $k$.

1. If $k$ is a number field, then $\operatorname{Exc}_{\text {dio }}^{1}(X) \neq \mathbb{P}\left(T_{X}\right)$ if and only if $X$ is not special.

2. If $k=\mathbb{C}$, then $\operatorname{Exc}_{\text {hol }}^{1}(X) \neq \mathbb{P}\left(T_{X}\right)$ if and only if $X$ is not special.

In the previous example, $X=\mathcal{C} \times \mathbb{P}^{1}$ with $\mathcal{C}$ a hyperbolic curve, we see that for every number field $L$ the closed subvariety $Y_{L}$ (the closure of $X(L)$ ) is the union of finitely many rational curves, corresponding to the fibres of $\mathrm{pr}_{1}=c_{X}$ over the $L$-rational points of $\mathcal{C}$. Thus $\operatorname{Exc}_{\text {dio }}^{1}(X)=\mathbb{P} \operatorname{pr}_{2}^{*}\left(T_{\mathbb{P}^{1}}\right)$, and in particular $\operatorname{Exc}_{\text {dio }}^{1}(X) \neq \mathbb{P}\left(T_{X}\right)$.

If $X$ is a closed subvariety of an abelian variety, then Conjecture 2 holds by Faltings' proof of Mordell and Lang [Fal94] and the work of Ueno, Yamanoi [Yam15] and Bloch, Ochiai and Kawamata [Kaw80] on closed subvarieties of abelian varieties.

Comparing Conjectures 1 and 2 suggests interesting questions. In particular, a strong uniform degeneracy statement in $\mathbb{P}\left(T_{X}\right)$ should imply a nondensity statement in $X$.

Conjecture 3. Let $X$ be a projective variety defined over a field $k$.

1. If $k$ is a number field and $\operatorname{Exc}_{\text {dio }}^{1}(X)=\mathbb{P}\left(T_{X}\right)$, then $X(k)$ is potentially dense.

2. If $k=\mathbb{C}$ and $\operatorname{Exc}_{\text {hol }}^{1}(X) \neq \mathbb{P}\left(T_{X}\right)$, then entire curves $f: \mathbb{C} \rightarrow X$ are algebraically degenerate - that is, the images $f(\mathbb{C})$ are not Zariski dense.

Interestingly, some examples of this phenomenon have already been proved in the foliated setting. If one considers a complex projective manifold $X$ equipped with a holomorphic (singular) foliation $\mathcal{F}$ such that all entire curves $f: \mathbb{C} \rightarrow X$ are tangent to this foliation, then it is proved in [McQ98] that if $X$ is a surface of general type and $\mathcal{F}$ is a foliation by curves, then entire curves are indeed algebraically degenerate. More recently, the same conclusion is shown in [BRT19] if $\mathcal{F}$ is a transversely hyperbolic foliation of codimension 1 and $X$ an arbitrary complex projective manifold $X$ (not necessarily of general type!).

\subsection{Function fields}

Given the well-known analogy between number fields and function fields, we formulate the foregoing conjectures in the function-field setting. Let $\kappa$ be an algebraically closed field of characteristic $0, \mathcal{C}$ be a smooth complex projective curve and $\kappa(\mathcal{C})$ its function field over $\kappa$. Let $X$ be a proper variety defined over $\kappa(\mathcal{C})$ and let $g: \mathcal{X} \rightarrow \mathcal{C}$ be the fibration associated to a proper model of $X$ over $\mathcal{C}$. In this setting, $\kappa(\mathcal{C})$-rational points correspond to sections $s: \mathcal{C} \rightarrow \mathcal{X}$ of $g$, and points defined over a finite extension $\kappa\left(\mathcal{C}^{\prime}\right) \supset \kappa(\mathcal{C})$ correspond to sections of the base change $X^{\prime}:=X \times_{\mathcal{C}} \mathcal{C}^{\prime} \rightarrow \mathcal{C}^{\prime}$ via a cover $\mathcal{C}^{\prime} \rightarrow \mathcal{C}$. We say that $X$ is isotrivial if there exists a model $\mathcal{X}$ over $\mathcal{C}$ and a cover $\mathcal{C}^{\prime} \rightarrow \mathcal{C}$ such that $\mathcal{X}^{\prime}$ is birational to $F \times \mathrm{C}^{\prime}$, where $F$ is a manifold.

In this setting, Lang's conjecture (in its weak version) can be formulated in the following way (see [Lan86, Historical appendix: algebraic families] for a historical discussion).

Conjecture 4. Let $X$ be nonisotrivial. If $X$ is of general type, then for all finite extensions $\kappa\left(\mathcal{C}^{\prime}\right) \supset \kappa(\mathcal{C})$, $\kappa\left(\mathcal{C}^{\prime}\right)$-points in $X$ are not Zariski dense. 
Special cases of Conjecture 4 have been proved in the literature. The analogue of Mordell's conjecture over function fields was proved by Manin [Man63] and Grauert [Gra65]. A higher-dimensional version is obtained in [Nog82] in the case of an ample cotangent bundle.

Similarly, the generalisation of Conjecture 1 is formulated as follows:

Conjecture 5. Let $X$ be nonisotrivial. Then $X$ is not special if and only if for all finite extensions $\kappa\left(\mathrm{C}^{\prime}\right) \supset \kappa(\mathrm{C}), \kappa\left(\mathrm{C}^{\prime}\right)$-points in $X$ are not Zariski dense.

Lang's stronger conjecture predicts the existence of an exceptional set, and moreover that such a set should be independent of the field of definition of the points.

Remark 3.3. If one wants to formulate a strong version of Conjecture 4 analogous to Lang's conjecture for varieties of general type over number fields, nonisotriviality is not enough, as shown by the example of a product of an isotrivial variety with a nonisotrivial one. One has to add some assumption on the nondensity of the subvarieties which are dominated by isotrivial varieties. Such a strong conjecture is considered in [Cam11, Conjecture 13.21].

Like in the number-field case, a stronger form of Conjecture 4 cannot be translated directly to nonspecial varieties. Instead we propose the following analogue of Conjecture 2 in the function-field setting:

Conjecture 6. Let $X$ be nonisotrivial. $X$ is not special if and only if for all finite extensions $\kappa\left(\mathfrak{C}^{\prime}\right) \supset$ $\kappa(\mathcal{C})$ there is a proper algebraic subset $Z \subset \mathbb{P}\left(T_{X}\right)$ such that sections $s: \mathcal{C}^{\prime} \rightarrow X^{\prime}$ whose liftings $s_{1}: \mathcal{C}^{\prime} \rightarrow \mathbb{P}\left(T_{X}\right)$ are not contained in $Z$ are finite.

In the number-field case, conjectures by Vojta (see [Voj87, Conjecture 3.4.3] and [Voj11, Conjecture 24.3]) predict a height bound for rational points in varieties of general type outside of the exceptional locus, which implies a nondensity statement.

In the function-field setting, a height bound is also expected, which is the content of the Lang-Vojta conjecture.

Conjecture 7. If $X$ is of general type and $\mathcal{L}$ is an ample line bundle on $X$, then there exist a proper algebraic subset $Z \subsetneq X$ and a positive constant $\alpha$ such that sections $s: \mathcal{C} \rightarrow X$ not contained in $Z$ (the model of Z) satisfy the inequality $\operatorname{deg} s^{*} \mathcal{L} \leq \alpha(2 g(\mathcal{C})-2)$, with $\mathcal{L}$ a model of $L$.

The isotrivial case of Conjecture 7 is known for subvarieties of abelian varieties [Yam15]. For certain cases of Conjecture 7 in the logarithmic setting, see [CZ08, CZ13, Tur17, ADT20, CT19].

One should notice that for function fields, the height bound predicted by Conjecture 7 can only imply a nondensity result when the variety is not isotrivial. We note that in the isotrivial case, one can expect that a natural generalisation of Conjecture 7 predicts the following:

Conjecture 8. If $(X, \Delta)$ is an orbifold of general type, then $(X, \Delta)$ is pseudo-algebraically hyperbolic as in Definition 2.11.

Motivated by Campana's core construction, already discussed, we propose the following extension of Conjecture 7 to nonspecial varieties:

Conjecture 9. If $X$ is not special, then there are a rational dominant map $\pi: X \rightarrow Y$ with $\operatorname{dim} Y>0$, a proper algebraic subset $Z \subsetneq \mathbb{P}\left(T_{X}\right)$, a positive constant $\alpha$ and an ample line bundle $L$ on $Y$ such that sections $s: \mathcal{C} \rightarrow X$, whose liftings $s_{1}: \mathcal{C} \rightarrow \mathbb{P}\left(T_{X}\right)$ are not contained in $Z$, satisfy the inequality $\operatorname{deg}(\pi \circ s)^{*} \mathcal{L} \leq \alpha(2 g(\mathcal{C})-2)$.

Finally, in the isotrivial case, a natural generalisation of Conjecture 9 to the logarithmic case predicts the following:

Conjecture 10. If $(X, D)$ is not special, then there are a rational dominant map $\pi:(X, D) \rightarrow\left(Y, D^{\prime}\right)$ with $\operatorname{dim} Y>0$, a proper algebraic subset $Z \subsetneq \mathbb{P}\left(T_{X}\right)$, a positive constant $\alpha$ and an ample line bundle 
$L$ on $Y$ such that sections $s: \mathcal{C} \rightarrow X$, whose liftings $s_{1}: \mathcal{C} \rightarrow \mathbb{P}\left(T_{X}\right)$ are not contained in $Z$, satisfy the inequality $\operatorname{deg}(\pi \circ s)^{*} \mathcal{L} \leq \alpha\left(2 g(\mathcal{C})-2+N_{\pi \circ s}^{[1]}\left(D^{\prime}\right)\right)$.

\section{Special versus weakly special}

In this section we construct a family of examples of varieties that are weakly special but are not special in the sense of Definition 2.3.

\subsection{Weakly special varieties}

We first recall the definition of weakly special varieties (the terminology is due to Campana).

Definition 4.1. A smooth projective variety $X$ over a field $k$ is weakly special if for every finite étale morphism $u: X^{\prime} \rightarrow X_{k}$, the variety $X^{\prime}$ does not admit a dominant rational map $f^{\prime}: X^{\prime} \rightarrow Z^{\prime}$ to a positive-dimensional variety $Z^{\prime}$ of general type. A projective variety $X$ is weakly special if some (hence any) desingularisation is weakly special.

The Weak Specialness Conjecture, whose original idea is linked to Abramovich and Colliot-Thélène in [HT00], predicts that weak specialness characterises potential density of rational points for varieties defined over number fields.

Conjecture 11 (see [HT00, Conjecture 1.2]). Let X be a projective variety defined over a number field. Then the set of rational points on $X$ is potentially dense if and only if $X$ is weakly special.

Conjecture 11 is still open: in fact, there is no example of a weakly special variety whose rational points are not potentially dense. The following conjecture is the analogue of Conjecture 11 for entire curves and function fields:

Conjecture 12. Let $X$ be a projective variety defined over $\mathbb{C}$. Then $X$ is weakly special if and only if

1. there exists an entire curve $\mathbb{C} \rightarrow X$ with Zariski dense image and

2. there are no dominant map $\pi: X \rightarrow Y$, ample line bundle $\mathcal{L}$, positive constant $\alpha$ and proper closed subset $Y_{\text {exc }}$ such that for every smooth integral curve $\mathcal{C}$ and morphism $s: \mathcal{C} \rightarrow X$ such that $s(\mathcal{C})$ is not contained in $Y_{\text {exc }}$, the following holds:

$$
\operatorname{deg}(\pi \circ s)^{*} \mathcal{L} \leq \alpha(2 g(\mathcal{C})-2) .
$$

As seen in Section 2, if a variety $X$ is special, then $X$ is weakly special. However, the two notions are equivalent only for curves and surfaces. We construct here examples of 3-dimensional projective varieties which are weakly special but not special. In Section 6 we will show that these examples contradict Conjecture 12.

\subsection{Examples of weakly special but not special threefolds}

The construction to come is a slight extension of the construction in [BT04] and was explained to us by Campana. We shall construct simply connected smooth projective threefolds $X$ having no rational fibrations onto varieties of general type but having equidimensional (elliptic) fibrations of (orbifold) general type $F: X \rightarrow S$ onto smooth surfaces $S$. These are thus examples of weakly special but not special varieties in the lowest dimension where the two notions do not agree.

Theorem 4.2. Let $m$ be a positive integer and let $T$, S be two surfaces together with fibrations $f: T \rightarrow \mathbb{P}^{1}$ and $g: S \rightarrow \mathbb{P}^{1}$ such that

1. $T$ is a smooth surface and the fibration $f: T \rightarrow \mathbb{P}^{1}$ has a single multiple fibre $f^{-1}(0)=: m \cdot T_{0}$, with $T_{0}$ a smooth elliptic curve, and another (singular) simply connected fibre; and 
2. $S$ is a smooth surface and the fibration $g: S \rightarrow \mathbb{P}^{1}$ has a smooth fibre $S_{0}:=g^{-1}(0)$ such that

(a) the surface $S$ is not of general type but the orbifold surface

$$
(S, \Delta):=\left(S,(1-1 / m) \cdot S_{0}\right)
$$

is of general type and

(b) the complement of $S_{0}$ in $S$ is simply connected.

Furthermore, let $X$ be the normalisation of the total spaces of the natural (orbifold) elliptic fibrations with equidimensional fibres defined by $f$ and $g-$ that $i$,

$$
X:=\left(S \times_{\mathbb{P}^{1}} T\right)^{v} \stackrel{F}{\longrightarrow}(S, \Delta) .
$$

Then $X$ is a weakly special projective smooth threefold that is not special.

Proof. We first notice that the orbifold base of the fibration $F$ is indeed $\Delta$ : by construction, the only multiple fibres lie above $S_{0}$, and $F^{*} S_{0}=m \cdot\left(S_{0} \times T_{0}\right)$. Since by assumption $(S, \Delta)$ is a surface of general type, it follows immediately that $X$ is not of special type.

We now prove that $X$ is weakly special. A key feature of $X$ in this direction is simple connectedness. Let $D:=F^{-1}\left(S_{0}\right)$; then the fibration $F:(X \backslash D) \rightarrow\left(S \backslash S_{0}\right)$ is a fibration without multiple fibres (since $f:\left(T \backslash T_{0}\right) \rightarrow \mathbb{P}^{1} \backslash\{0\}$ has no multiple fibres), and with a simply connected fibre. This implies that $F_{*}: \pi_{1}(X \backslash D) \rightarrow \pi_{1}\left(S \backslash S_{0}\right)$ is an isomorphism. Using our assumption, the group $\pi_{1}(X \backslash D)$ is thus trivial. This implies that $X$ is simply connected, since the natural map $\pi_{1}(X \backslash D) \rightarrow \pi_{1}(X)$ is surjective.

As a consequence, to prove that $X$ is weakly special it suffices to show that no fibration $h: X \rightarrow Z$ exists, with $Z$ of general type and of dimension $d$, with $0<d \leq 3$, since $X$ does not admit any nontrivial étale cover. Assume by contradiction that such an $h$ exists. Then $d<3$, because $X$ is not of general type, since it is an elliptic fibration over $S$; hence $d=1,2$. Note that since $X$ is simply connected, $Z$ has to be simply connected, and thus $d>1$, since the only simply connected curve is $\mathbb{P}^{1}$, which is not of general type. We are reduced to the case in which $d=2$, and $h \neq F$, since by assumption $S$ is not of general type. Since by construction the fibres of $F$ are special, it follows from [Cam04, Theorem 2.7] that there exists a map $g: S \rightarrow Z$. But since $S$ is not of general type, this contradicts the assumption that $Z$ is a surface of general type.

We now give examples of fibrations $T \rightarrow \mathbb{P}^{1}$ and $S \rightarrow \mathbb{P}^{1}$ that satisfy the hypothesis of Theorem 4.2. In particular, the examples show that such fibrations exist for every $m \geq 2$ and therefore provide examples of countably many weakly special threefolds $X$ as a corollary of Theorem 4.2.

Example 4.3 (First fibration). In order to construct $f: T \rightarrow \mathbb{P}^{1}$, we consider first a fibration $f^{\prime}: T^{\prime} \rightarrow \mathbb{P}^{1}$ of an elliptic surface having no multiple fibre, a smooth fibre $T_{0}^{\prime}:=\left(f^{\prime}\right)^{-1}(0)$ and a (singular) simply connected fibre, and moreover such that $p_{g}\left(T^{\prime}\right):=h^{0,2}\left(T^{\prime}\right)=0$. Then $T^{\prime}$ is simply connected. Let $m>1$ be an integer. The Kodaira logarithmic transform $f: T \rightarrow \mathbb{P}^{1}$ of order $m$ on the fibre $T_{0}^{\prime}$ of $f^{\prime}$ is a new elliptic fibration which replaces $T_{0}^{\prime}$ by a smooth multiple fibre $m \cdot T_{0}$ of multiplicity $m$ over $0 \in \mathbb{P}^{1}$, leaving the complements $T^{\prime} \backslash T_{0}^{\prime}$ and $T \backslash m \cdot T_{0}$ isomorphic as elliptic fibrations over $\mathbb{P}^{1} \backslash\{0\}$ (see, for example, [BT04, Section 3], and for more details, [FM94, Section 1.6]). Moreover, $p_{g}(T)=p_{g}\left(T^{\prime}\right)=0$, and $T$ is still simply connected since $f$ still has a simply connected fibre. This implies that $b_{1}(T)=0$ is even and $T$ is Kähler; since $p_{g}(T)=0$, we conclude that $T$ is projective (we refer to [FM94, Section 1.6] for the details).

In the original construction of [BT04], $S$ was chosen so that $\kappa(S)=1$. We shall see that a small variation of the construction permits us to choose for $\left(S, \Delta_{F}\right)$ a suitable blowup of any smooth projective surface $S^{\prime}$ with $\kappa\left(S^{\prime}\right) \in\{-\infty, 0,1\}$ and $\Delta_{F}$ the strict transform of a suitable ample orbifold $\mathbb{Q}$-divisor on $S^{\prime}$. 
Example 4.4 (Second fibration). In order to construct $g: S \rightarrow \mathbb{P}^{1}$ (given $m$ ), we consider a simply connected smooth projective surface $S^{\prime}$ that is not of general type, together with an ample and smooth divisor $C^{\prime}$, which is a member of a pencil $C_{t}^{\prime}$ of divisors on $S^{\prime}$ (whose generic member $C^{\prime \prime}$ meets $C^{\prime}$ transversally at $C^{\prime 2}$ distinct points), such that the complete linear system $\mathcal{O}_{S^{\prime}}\left(C^{\prime}\right)$ is base-point free and $K_{S^{\prime}}+(1-1 / m) \cdot C^{\prime}$ is a big divisor on $S^{\prime}$.

For example, we can choose $S^{\prime}=\mathbb{P}^{2}$, together with $C^{\prime}$ a smooth curve of degree $d \geq 4$ if $m \geq 5$, and $d \geq 7$ if $m \geq 2$.

Now choose a second generic member $C^{\prime \prime}$ of the linear system $\left|C^{\prime}\right|$ meeting $C^{\prime}$ transversally at $\left(C^{\prime}\right)^{2}$ distinct points. Let $\sigma: S \rightarrow S^{\prime}$ be the blowup of $S^{\prime}$ at these $\left(C^{\prime}\right)^{2}$ points, let $\Delta$ be the strict transform of $\Delta^{\prime}:=(1-1 / m) \cdot C^{\prime}$ on $S$ and let $E$ be the exceptional divisor of $\sigma$. Then the divisor

$$
K_{S}+\Delta=\left(\sigma^{*} K_{S^{\prime}}+E\right)+\left(\sigma^{*} \Delta^{\prime}-(1-1 / m) \cdot E\right)=\sigma^{*}\left(K_{S^{\prime}}+\Delta^{\prime}\right)+(1 / m) \cdot E
$$

is big, since $\sigma$ is birational and all the varieties are projective; this implies that the orbifold surface $(S, \Delta)$ is of general type.

Let $k>0$ be the order of divisibility of $\left[C^{\prime}\right]$ in $\operatorname{Pic}\left(S^{\prime}\right)$. By (a version of) the Lefschetz theorem, $\pi_{1}\left(S^{\prime} \backslash C^{\prime}\right)$ is the cyclic group of order $k$ generated by a small loop around $C^{\prime}$. But blowing up a point $a$ on $C^{\prime}$ makes this loop become trivial in $\pi_{1}\left(E_{a} \backslash\{a\}\right)=\pi_{1}(\mathbb{C})=0$, where $E_{a} \cong \mathbb{P}^{1}$ is the exceptional divisor of the blowup over $a$, and $a \in C^{\prime} \cap C^{\prime \prime} \subset S^{\prime}$. This loop thus becomes homotopically trivial in $\left(S \backslash S_{0}\right)$, which is thus simply connected.

Remark 4.5. The smoothness of $C^{\prime}$ is not necessary. One may just assume that $C^{\prime}$ is nodal and that $C^{\prime \prime}$ meets $C^{\prime}$ at smooth points of $C^{\prime}$.

\section{Degeneracy results for surfaces}

In this section we extend results of Corvaja and Zannier in [CZ04] for number fields to the orbifold setting, both over function fields and in Nevanlinna theory. We prove hyperbolicity and degeneracy results for a class of surfaces as a combination of ideas of Corvaja and Zannier together with the recent method introduced by Ru and Vojta in [RV20].

In particular we recover the function-field and analytic analogues of [CZ04, Main Theorem] in the logarithmic setting. We stress that even if the arithmetic result for orbifold rational points seems at the moment out of reach, our results give evidence for the arithmetic part of Conjecture 1. Finally, all these results will be applied in Section 6 to the nonspecial threefold $X_{m}$ defined in Section 4.

\subsection{Function fields}

In this section we let $\kappa$ be an algebraically closed field of characteristic 0 and we assume all the varieties defined over $\kappa$. The main theorem of this section will be a consequence of the following statement, whose proof is contained in Section 7 (see Theorem 7.6). We start by recalling the definition of the $\beta$ invariant (we refer to [RV20] for a discussion and properties).

Definition 5.1. Let $X$ be a complete variety, let $\mathcal{L}$ be a big line bundle on $X$ and let $D$ be a nonzero effective Cartier divisor on $X$. We define

$$
\beta(\mathcal{L}, D)=\liminf _{N \rightarrow \infty} \frac{\sum_{m \geq 1} h^{0}\left(X, \mathcal{L}^{N}(-m D)\right)}{N h^{0}\left(X, \mathcal{L}^{N}\right)} .
$$

Using the constant $\beta$ we can reformulate the main theorem of [RV20] in the function-field case. However, for our applications we need to obtain an explicit dependence of the constants on the Euler characteristic of the curve. This is obtained in the following theorem, which we state in the constant case, by use of the more explicit [Wan04, Theorem 1] instead of the function-field analogue of the Schmidt subspace theorem [Wan04, Main Theorem]: 
Theorem 5.2. Let $X \subset \mathbb{P}^{m}$ be a projective variety over $\kappa$ of dimension $n$, let $D_{1}, \ldots, D_{q}$ be effective Cartier divisors intersecting properly on $X$ and let $\mathcal{L}$ be a big line sheaf. Let $\mathcal{C}$ be a smooth projective curve over $\kappa$, let $S$ be a finite set of points on $\mathrm{C}$ and let $K=\kappa(\mathrm{C})$ be the function field of $\mathrm{C}$. Then for any $\epsilon>0$, there exist constants $c_{1}$ and $c_{2}$, independent of the curve $\mathcal{C}$ and the set $S$, such that for any map $x=\left[x_{0}: \ldots: x_{m}\right]: \mathcal{C} \rightarrow X$, where $x_{i} \in K$, outside the augmented base locus of $\mathcal{L}$ either

$$
\sum_{i=1}^{q} \beta\left(\mathcal{L}, D_{i}\right) m_{D_{i}, S}(x) \leq(1+\epsilon) h_{\mathcal{L}}(x)+c_{1} \max \{1,2 g(\mathcal{C})-2+|S|\},
$$

or the image of $x$ is contained in a hypersurface (over $\kappa)$ in $\mathbb{P}^{m}$ of degree at most $c_{2}$.

\section{Remark 5.3.}

1. In the case where $X$ is nonsingular, the condition on the proper intersection is equivalent to general position. We refer to [RV20, Definition 2.1] for precise statements and properties.

2. Even if Theorem 5.2 is stated in the split case, we note that our proof carries over to the nonsplit case almost verbatim. We focus here only on the split case because it is the one relevant for our applications.

3. The constants $c_{1}$ and $c_{2}$ in Theorem 5.2 can be effectively computed given $X, D_{i}, \mathcal{L}, \varepsilon$, so in particular the algebraic hyperbolicity bounds can be made effective in the same way.

The proof of Theorem 5.2 is included in Section 7 as Theorem 7.6, and we refer to Section 7.2 for the definitions of all the quantities involved. For our application we will use the following corollary in dimension 2, whose proof is also included in Section 7 (see Corollary 7.7):

Corollary 5.4. In the previous setting, if $X$ has dimension 2 , then for any $\epsilon>0$, there exist constants $c_{1}$ and $c_{3}$ independent of the curve $\mathcal{C}$ and the set $S$ such that for any $K$-point $x=\left[x_{0}: \ldots: x_{m}\right]: \mathcal{C} \rightarrow X$, with $x_{i} \in K$, either $\operatorname{deg} x(\mathcal{C}) \leq c_{3}$ or

$$
\sum_{i=1}^{q} \beta\left(\mathcal{L}, D_{i}\right) m_{D_{i}, S}(x) \leq(1+\epsilon) h_{\mathscr{L}}(x)+c_{1} \max \{1,2 g(\mathcal{C})-2+|S|\} .
$$

We now state the main result of this section in the logarithmic case.

Theorem 5.5. Let $X \subset \mathbb{P}^{m}$ be a smooth projective surface and $D=D_{1}+\cdots+D_{r}$ be a divisor with $r \geq 2$, both defined over $\kappa$, such that

1. no three components $D_{i}$ meet at a point and

2. there exists a choice of positive integers $p_{i}$ such that

$\circ$ the divisor $D_{p}:=p_{1} D_{1}+p_{2} D_{2}+\cdots+p_{r} D_{r}$ is ample and

- The inequality

$$
2 D_{p}^{2} \xi_{i}>\left(D_{p} \cdot D_{i}\right) \xi_{i}^{2}+3 D_{p}^{2} p_{i}
$$

holds for every $i=1, \ldots, r$, where $\xi_{i}$ is the minimal positive solution of the equation $D_{i}^{2} x^{2}-2\left(D_{p} \cdot D_{i}\right) x+D_{p}^{2}=0$.

Then $(X, D)$ is pseudo-algebraically hyperbolic (see Definition 2.9).

The arithmetic analogue of Theorem 5.5 was proved by Corvaja and Zannier in [CZ04, Main Theorem]. Our proof is different from in the arithmetic case, because it relies on Theorem 5.2, whereas Corvaja and Zannier's method relies on a direct application of Schmidt's subspace theorem (and, as noted by Vojta, their proof does not apply directly to the function-field case). Nevertheless, we will use some of the techniques of [CZ04] in computing the constants $\beta$.

Lemma 5.6. In the same setting as Theorem 5.5, for every $i=1, \ldots, r, \beta_{i}=\beta\left(D_{p}, D_{i}\right)>p_{i}$. 
Proof. By the Riemann-Roch theorem, for $N$ large enough we have $2 h^{0}\left(N D_{p}\right)=D_{p}^{2} N^{2}+O(N)$. In order to compute $\beta_{i}$, we consider the divisor $N D_{p}-m D_{i}$, which is effective if $m \leq N p_{i}$.

By the same computation as in [CZ04] (in particular see pp. 718-719), we get

$$
\sum_{m=0}^{\xi_{i} N} h^{0}\left(N D_{p}-m D_{i}\right) \geq N^{3}\left(\frac{\xi_{i}^{2}\left(D_{p} \cdot D_{i}\right)}{2}-\frac{\xi_{i}^{3} D_{i}^{2}}{3}\right)+O\left(N^{2}\right)
$$

Recall that by the definition of $\xi_{i}$, we have $D_{i}^{2} \xi_{i}^{3}=2\left(D_{p} \cdot D_{i}\right) \xi_{i}^{2}-D_{p}^{2} \xi=0$. Using this in formula (5.1), we obtain

$$
\begin{aligned}
2 \sum_{m=0}^{\xi_{i} N} h^{0}\left(N A-m D_{i}\right) & \geq 2 N^{3}\left(\frac{\xi_{i}^{2}\left(D_{p} \cdot D_{i}\right)}{2}-\frac{2 \xi_{i}^{2}\left(D_{p} \cdot D_{i}\right)}{3}+\frac{D_{p}^{2} \xi_{i}}{3}\right)+O\left(N^{2}\right) \\
& =\left(\frac{2}{3} \xi_{i} D_{p}^{2}-\frac{1}{3}\left(D_{p} \cdot D_{i}\right) \xi_{i}^{2}\right) N^{3}+O\left(N^{2}\right) .
\end{aligned}
$$

This implies that

$$
\beta_{i}=\frac{\frac{2}{3} \xi_{i} D_{p}^{2}-\frac{1}{3}\left(D_{p} \cdot D_{i}\right) \xi_{i}^{2}}{D_{p}^{2}}>p_{i}
$$

We also include an easy lemma which provides a useful trick to get a lower bound of the height of a point with respect to $D_{p}$.

Lemma 5.7. In the same setting as Theorem 5.5, let $\varepsilon=\min \left\{\left(\beta-p_{i}\right) / p_{i}\right\}$; then for every $\varphi: \mathcal{C} \rightarrow X$,

$$
\sum_{i=1}^{r} \beta_{i} h_{D_{i}}(\varphi) \geq(1+\varepsilon) h_{D_{p}}(\varphi)
$$

Proof. We have that for every $i$,

$$
\beta_{i} h_{D_{i}}(\varphi)=\left(\frac{\beta_{i}-p_{i}}{p_{i}}+1\right) p_{i} h_{D_{i}}(\varphi)
$$

Summing over $i=1, \ldots, r$, we get

$$
\sum_{i=1}^{r} \beta_{i} h_{D_{i}}(\varphi)=\sum_{i=1}^{r}\left(\frac{\beta_{i}-p_{i}}{p_{i}}+1\right) p_{i} h_{D_{i}}(\varphi) \geq(1+\varepsilon) h_{D_{p}}(\varphi) .
$$

Proof of Theorem 5.5. We will prove that there exists a constant $\alpha>0$ such that for every morphism $\varphi: \mathcal{C} \rightarrow X$ with $\varphi(\mathcal{C}) \nsubseteq D$, we have

$$
\operatorname{deg} \varphi^{*} D_{p} \leq \alpha \cdot \max \left\{1,2 g(\mathcal{C})-2+N_{\varphi}^{[1]}\left(D_{p}\right)\right\}
$$

We note that such a bound implies that $(X, D)$ is pseudo-algebraically hyperbolic in the sense of Definition 2.9, provided that the union of images of curves for which $2 g(\mathcal{C})-2+N_{\varphi}^{[1]}\left(D_{p}\right) \leq 0$ is a proper closed subset of $X$. We will first prove the bound (5.2) and then show that the exceptional set is indeed a proper and closed subset. First we note that condition (1) of the theorem implies that $D_{1}, \ldots, D_{r}$ are in general position. We fix 


$$
\varepsilon=\min _{i}\left\{\frac{\beta_{i}-p_{i}}{p_{i}}\right\},
$$

which is positive by Lemma 5.6. Let $\varphi: \mathcal{C} \rightarrow X$ be a morphism such that $\varphi(\mathcal{C}) \nsubseteq D$. By Corollary 5.4, applied with $\mathcal{L}=\mathcal{O}_{X}\left(D_{p}\right)$, there exist constants $c_{1}$ and $c_{3}$ depending only on $X$ and $\varepsilon$ (independent of $\mathcal{C}$ and $\varphi$ ) such that either $\varphi(\mathcal{C})$ has degree bounded by $c_{3}$ or

$$
\sum_{i=1}^{r} \beta_{i} m_{D_{i}, S}(\varphi) \leq\left(1+\frac{\epsilon}{2}\right) h_{D_{p}}(\varphi)+c_{2} \max \{1,2 g(\mathcal{C})-2+|S|\},
$$

where $S=\operatorname{supp}\left(\varphi^{*} D\right)$. In the first case we are done. In the latter, since the support of $\varphi^{*} D$ is contained in $S, m_{D_{i}, S}(\varphi)=h_{D_{i}}(\varphi)+O(1)$.

Lemma 5.7 gives the lower bound

$$
\sum_{i=1}^{r} \beta_{i} m_{D_{i}, S}(\varphi)>(1+\epsilon) h_{D_{p}}(\varphi) .
$$

Together with formula (5.3), this implies that there exists a constant $c_{2}^{\prime}$ such that

$$
\frac{\epsilon}{2} h_{D_{p}}(\varphi)<c_{2}^{\prime} \max \{1,2 g(\mathcal{C})-2+|S|\}
$$

Hence,

$$
\operatorname{deg} \varphi^{*} D_{p}=h_{D_{p}}(\varphi)<2 c_{2}^{\prime} \epsilon^{-1} \max \left\{1,2 g(\mathcal{C})-2+N_{\varphi}^{[1]}\left(D_{p}\right)\right\}
$$

To finish the proof we need to show that there exists a closed subvariety $Z$ that contains all images $\varphi(\mathcal{C})$ when $2 g(\mathcal{C})-2+N_{\varphi}^{[1]}\left(D_{p}\right)$ is not positive. First of all, we can reduce to the case in which $\kappa=\mathbb{C}$ : it is enough to notice that given $(X, D)$ defined over $\kappa$, there exists a field $\kappa_{0}$ that is an algebraically closed subfield of $\kappa$ which is finitely generated over $\overline{\mathbb{Q}}$ and such that $(X, D)$ has a model over $\kappa_{0}$. For such a $\kappa_{0}$ there exists an embedding $\kappa_{0} \rightarrow \mathbb{C}$, and therefore we can reduce the problem to the case in which $(X, D)$ is defined over $\mathbb{C}$.

In this case, we claim that the exceptional set $Z$ can be chosen to be the exceptional set in [Lev09, Theorem $8.3 \mathrm{~B}$ ]. In fact, Levin's same strategy applies to the setting of our theorem (in particular, the proof of [CZ04, Main Theorem] shows that $D_{p}$ is large in Levin's sense), and therefore there exists a proper closed subvariety $Z \subset X$ that contains all the images of entire curves $\mathbb{C} \rightarrow X$. To conclude, it is sufficient to notice that for every curve $\mathcal{C}$ such that $2 g(\mathcal{C})-2+N_{\varphi}^{[1]}\left(D_{p}\right) \leq 0$, there exists a nontrivial holomorphic map $\mathbb{C} \rightarrow \mathcal{C} \backslash \operatorname{supp} \varphi^{*} D_{p}$. In particular, this implies that $\varphi(\mathcal{C})$ has to be contained in $Z$, as desired.

Next we show that Theorem 5.5 leads to a statement for orbifold morphisms; in particular, we obtain the result that Conjecture 8 holds in this setting.

Corollary 5.8. Let $(X, D)$ be as in Theorem 5.5. Let $\Delta$ be the $\mathbb{Q}$-divisor defined as

$$
\Delta=\sum_{i=1}^{r}\left(1-\frac{1}{m_{i}}\right) D_{i}
$$

for some integers $m_{i} \geq 1$. Then there exists a positive integer $m>0$ such that if $m_{i}>m$ for every $i$, $(X, \Delta)$ is pseudo-algebraically hyperbolic (see Definition 2.11).

We obtain the previous corollary by applying the following general lemma to Theorem 5.5. Note that given an orbifold $(X, \Delta)$ such that $X \backslash \operatorname{supp}(\Delta)$ is of log general type, there exists an integer $m>0$ such that if all orbifold multiplicities of $\Delta$ are greater than $m$, the orbifold $(X, \Delta)$ is of general type. In particular, the following lemma is in accordance with Conjecture 8: 
Lemma 5.9. Let $(X, \Delta)$ be an orbifold defined over $\kappa$ such that $(X, \operatorname{supp}(\Delta))$ is pseudo-algebraically hyperbolic with a constant $\alpha>0$ and with exceptional set $Z \subset X$. Then there exists a positive integer $m>0$ such that if all orbifold multiplicities are greater than $m,(X, \Delta)$ is pseudo-algebraically hyperbolic with the same constant $\alpha$ and the same exceptional set $Z$.

We note that Lemma 5.9 and its proof generalise [Rou10, Theorem 4.8], where it was assumed that $X=\mathbb{P}^{n}$.

Proof of Lemma 5.9. Let $\psi:\left(\mathcal{C}, \Delta_{C}\right) \rightarrow(X, \Delta)$ be an orbifold morphism. We will use the notation $\delta:=N_{\psi}^{[1]}(\operatorname{supp}(\Delta))$. Let

$$
\begin{aligned}
\psi^{*}\left(\Delta_{j}\right) & =\sum_{i=1}^{\delta} t_{i, j} P_{i}, \\
\psi^{*}(\operatorname{supp}(\Delta)) & =\sum_{i=1}^{\delta} t_{i} P_{i},
\end{aligned}
$$

where the $P_{i}$ are the distinct points of $\psi^{-1}(\operatorname{supp}(\Delta))$. Then if

$$
\Delta_{C}=\sum_{i=1}^{\delta}\left(1-\frac{1}{m_{i}^{\prime}}\right) P_{i}
$$

$\psi$ is an orbifold morphism if $m_{i}^{\prime} t_{i} \geq m_{j}$ for all $j \in \varphi(i)$, where $\varphi(i)$ is the set

$$
\varphi(i):=\left\{k: 1 \leq k \leq \delta \text { and } \psi\left(P_{i}\right) \in \Delta_{k}\right\} .
$$

We define the orbifold structure $\widetilde{\Delta}$ on $\mathcal{C}$ by the multiplicities

$$
\widetilde{m_{i}}=\sup _{j \in \varphi(i)}\left\lceil\frac{m_{j}}{t_{i}}\right\rceil,
$$

where, as usual, $\lceil k\rceil$ denotes the rounding up of $k$. By definition, $\psi:(C, \widetilde{\Delta}) \rightarrow(X, \Delta)$ is an orbifold morphism. Moreover, for every orbifold structure $\Delta^{\prime}$ on $\mathcal{C}$ such that $\psi:\left(C, \Delta^{\prime}\right) \rightarrow(X, \Delta)$ is an orbifold morphism, we have $\Delta^{\prime} \geq \widetilde{\Delta}$. In particular, it is enough to prove that $\psi$ satisfies a degree bound as in Definition 2.11 for the orbifold structure $\widetilde{\Delta}$. In other words, we have to prove that there exists a constant $\alpha_{\Delta}$ and an ample line bundle $\mathcal{L}_{\Delta}$ on $X$ such that for every $\psi$ such that $\psi(\mathcal{C}) \nsubseteq Z$, we have

$$
\operatorname{deg} \psi^{*} \mathcal{L}_{\Delta} \leq \alpha_{\Delta}\left(2 g(C)-2+\sum_{i=1}^{\delta}\left(1-\frac{1}{\widetilde{m_{i}}}\right)\right) .
$$

We can bound the contribution coming from the orbifold divisor as follows:

$$
\sum_{i=1}^{\delta}\left(1-\frac{1}{\bar{m}_{i}}\right) \geq \sum_{i=1}^{\delta}\left(1-\frac{t_{i}}{\sup _{j \in \varphi(i)} m_{j}}\right) \geq N_{\psi}^{[1]}(\operatorname{supp}(\Delta))-\sum_{i=1}^{\delta} \sum_{j=1}^{q} \frac{t_{i, j}}{m_{j}}
$$

On the other hand, by definition,

$$
\sum_{i=1}^{\delta} t_{i, j}=\operatorname{deg}\left(\psi^{*} \Delta_{j}\right)
$$


which implies that we can rewrite formula (5.4) as

$$
\sum_{i=1}^{\delta}\left(1-\frac{1}{\bar{m}_{i}}\right) \geq N_{\psi}^{[1]}(\operatorname{supp}(\Delta))-\sum_{j=1}^{q} \frac{\operatorname{deg}\left(\psi^{*} \Delta_{j}\right)}{m_{j}} .
$$

In particular, formula (5.5) can be rewritten as

$$
N_{\psi}^{[1]}(\operatorname{supp}(\Delta)) \leq \sum_{i=1}^{\delta}\left(1-\frac{1}{\widetilde{m_{i}}}\right)+\sum_{j=1}^{q} \frac{\operatorname{deg}\left(\psi^{*} \Delta_{j}\right)}{m_{j}} .
$$

On the other hand, our assumption that $(X, \operatorname{supp}(\Delta))$ is pseudo-algebraically hyperbolic implies that for every ample line bundle $\mathcal{L}$ on $L$, if $\psi(\mathcal{C}) \nsubseteq Z$, we have

$$
\operatorname{deg} \psi^{*} \mathcal{L} \leq \alpha\left(2 g(\mathcal{C})-2+N_{\psi}^{[1]}(\operatorname{supp} \Delta)\right)
$$

Finally, combining formulas (5.7) and (5.6), we obtain

$$
\operatorname{deg} \psi^{*} \mathcal{L} \leq \alpha(2 g(\mathcal{C})-2+\operatorname{deg} \widetilde{\Delta})+\operatorname{deg} \psi^{*}\left(\sum_{j=1}^{q} \frac{\alpha}{m_{j}} \Delta_{j}\right)
$$

Therefore, we conclude by noticing that when the multiplicities $m_{j}$ are big enough, the line bundle

$$
\mathcal{L}_{\Delta}=\mathcal{L} \otimes \mathcal{O}_{X}\left(-\sum_{j=1}^{\delta} \frac{\alpha}{m_{j}} \Delta_{j}\right)
$$

is ample on $X$, and therefore we obtain

$$
\operatorname{deg} \psi^{*} \mathcal{L}_{\Delta} \leq \alpha(2 g(\mathcal{C})-2+\operatorname{deg} \widetilde{\Delta}) .
$$

Finally we can prove Corollary 5.8 .

Proof of Corollary 5.8. The conclusion follows from Theorem 5.5 and Lemma 5.9.

\subsection{Holomorphic maps}

In this subsection we are interested in the degeneracy properties of holomorphic maps. All varieties will be defined over $\mathbb{C}$. As before, we will obtain our result as a consequence of the following generalisation of [RV20, General Theorem (Analytic Part)] that includes truncation:

Theorem 5.10. Let $X$ be a complex projective variety of dimension $n$ and let $D_{1}, \ldots, D_{q}$ be effective Cartier divisors intersecting properly on $X$. Let $\mathcal{L}$ be a big line bundle. For each $\varepsilon>0$, there exists a positive integer $Q$ such that for any algebraically nondegenerate holomorphic map $f: \mathbb{C} \rightarrow X$, the inequality

$$
\sum_{j=1}^{q} \beta\left(\mathscr{L}, D_{j}\right) T_{D_{j}, f}(r)-(1+\varepsilon) T_{\mathscr{L}, f}(r) \leq_{\operatorname{exc}} \sum_{j=1}^{q} \beta\left(\mathscr{L}, D_{j}\right) N_{f}^{(Q)}\left(D_{j}, r\right)
$$

holds, where $\leq_{\mathrm{exc}}$ means that the inequality holds for all $r \in \mathbb{R}^{+}$except a set of finite Lebesgue measure. 
The proof of Theorem 5.10 will be given in Section 7 (see in particular Theorem 7.1). Using Theorem 5.10, we obtain a Nevanlinna analogue of Theorem 5.5 and Corollary 5.8.

Theorem 5.11. Let $X \subset \mathbb{P}^{m}$ be a complex nonsingular projective surface and $D=D_{1}+\cdots+D_{q}$ be a divisor with $q \geq 2$, such that

1. no three components $D_{i}$ meet at a point and

2. there exists a choice of positive integers $p_{i}$ such that

$\circ$ the divisor $D_{p}:=p_{1} D_{1}+p_{2} D_{2}+\cdots+p_{q} D_{q}$ is ample and

○ the inequality

$$
2 D_{p}^{2} \xi_{i}>\left(D_{p} \cdot D_{i}\right) \xi_{i}^{2}+3 D_{p}^{2} p_{i}
$$

holds for every $i=1, \ldots, q$, where $\xi_{i}$ is the minimal positive solution of the equation $D_{i}^{2} x^{2}-2\left(D_{p} \cdot D_{i}\right) x+D_{p}^{2}=0$.

Let $\Delta$ be the $\mathbb{Q}$-divisor defined as

$$
\Delta=\sum_{i=1}^{q}\left(1-\frac{1}{m_{i}}\right) D_{i}
$$

Then there exists a positive integer $m$ such that if $m_{i} \geq m$ for every $i$, every orbifold entire curve $\psi: \mathbb{C} \rightarrow(X, \Delta)$ is algebraically degenerate.

Proof. First we note that condition 1 implies that $D_{1}, \ldots, D_{q}$ are in general position. Let $f: \mathbb{C} \rightarrow(X, \Delta)$ be a Zariski dense orbifold entire curve. As before, we write $\beta_{i}=\beta\left(D_{p}, D_{i}\right)$ and we set

$$
\varepsilon=\min \left\{\left(\beta_{i}-p_{i}\right) / p_{i}\right\}
$$

which is positive by Lemma 5.6. By Theorem 5.10, applied with $\mathcal{L}=\mathcal{O}_{X}\left(D_{p}\right)$ and $\varepsilon / 2$, there exists an integer $Q$ such that the following inequality holds:

$$
\sum_{j=1}^{q} \beta_{j} T_{D_{j}, f}(r)-\left(1+\frac{\varepsilon}{2}\right) T_{D_{p}, f}(r) \leq \operatorname{exc} \sum_{j=1}^{q} \beta_{j} N_{f}^{(Q)}\left(D_{j}, r\right) .
$$

The analogue of Lemma 5.7 in the Nevanlinna setting gives the lower bound

$$
\sum_{j=1}^{q} \beta_{i} T_{D_{i}, f}(r)>(1+\varepsilon) T_{D_{p}, f}(r) .
$$

Together with formula (5.8), this implies that

$$
\frac{\epsilon}{2} T_{D_{p}, f}(r) \leq \sum_{j=1}^{q} \beta_{j} N_{f}^{(Q)}\left(D_{j}, r\right) .
$$

Since $f$ is an orbifold entire curve, we have

$$
N_{f}^{(Q)}\left(D_{j}, r\right) \leq \frac{Q}{m_{j}} N_{f}\left(D_{j}, r\right) \leq \frac{Q}{m_{j}} T_{D_{p}, f}(r) .
$$

Therefore, we can rewrite formula (5.9) as

$$
\frac{\epsilon}{2} T_{D_{p}, f}(r) \leq Q\left(\sum_{j=1}^{q} \frac{\beta_{j}}{m_{j}}\right)_{D_{p}, f}(r) \leq \frac{Q}{m}\left(\sum_{j=1}^{q} \beta_{j}\right) T_{D_{p}, f}(r) .
$$


To conclude, it is enough to choose $m$ big enough such that

$$
\frac{Q}{m}\left(\sum_{j=1}^{q} \beta_{j}\right)<\frac{\varepsilon}{2}
$$

As a corollary, we obtain the analogue of [CZ04, Main Theorem] and Theorem 5.5 in the Nevanlinna setting.

Corollary 5.12. Let $(X, D)$ be as in Theorem 5.11. Then every entire curve $\psi: \mathbb{C} \rightarrow X \backslash D$ is algebraically degenerate. Moreover, $(X, D)$ is Brody hyperbolic modulo the exceptional set $Z$ of Theorem 5.5 - that is, every entire curve $\psi: \mathbb{C} \rightarrow X \backslash D$ satisfies that $\psi(\mathbb{C})$ is contained in $Z$.

Proof. The first statement follows directly from Theorem 5.11. For the second statement we can directly apply [Lev09, Theorem 8.3 B] to our setting (since, as noted before, the proof of [CZ04, Main Theorem] shows that the divisor $D_{p}$ is large in Levin's sense).

\section{Degeneracy properties of $X_{m}$}

Given Lang and Vojta's dictionary between arithmetic and geometric properties of a variety $X$, it is expected that varieties with a potentially dense set of rational points should correspond to manifolds admitting Zariski dense entire curves. Therefore, the analogue of Conjecture 11 should imply that weakly special manifolds should admit such curves (see Conjecture 12).

Campana and Păun [CP07] have shown that some examples constructed in [BT04] (in particular with $\kappa(S)=1$ ) give counterexamples to such a statement. In other words, there are some weakly special manifolds in which all entire curves are algebraically degenerate. The goal of this section is to show that one can produce many more 'counterexamples' from the examples given in the previous section. In particular, we show that the weakly special varieties $X_{m}$ (for $m$ big enough) provide counterexamples to Conjecture 12.

\subsection{Construction}

Following ideas of Corvaja and Zannier in [CZ10], we begin by giving a series of examples in which the results of Section 5 apply. As in Section 5, we denote by $\kappa$ an algebraically closed field of characteristic 0 . We start with the following definition:

Definition 6.1. Let $D_{1}, D_{2}$ and $D_{3}$ be three projective plane curves in $\mathbb{P}_{K}^{2}$ and let $D_{4}=H$ be a hyperplane such that $D_{i} \cap D_{j} \cap D_{k}$ is empty for every distinct $i, j, k$. Given three distinct projective curves $B_{1}, B_{2}, B_{3}$, we say that $\left(D_{1}, B_{1}\right),\left(D_{2}, B_{2}\right),\left(D_{3}, B_{3}\right)$ and $H$ are in general position if

○ $D_{i}$ and $B_{i}$ intersect transversally for every $i=1,2,3$ and

○ $D_{i} \cap D_{j} \cap B_{h}=\emptyset$ for every distinct $i, j$ and $h \in\{i, j\}$.

The main source of examples will be certain blowups of $\mathbb{P}^{2}$. In the next proposition we show that these satisfy the assumptions of Theorems 5.5 and 5.11.

Proposition 6.2. Let $\left(D_{1}, B_{1}\right),\left(D_{2}, B_{2}\right),\left(D_{3}, B_{3}\right)$ and $H$ be curves in general position as in Definition 6.1, such that $\operatorname{deg} D_{i} \geq \operatorname{deg} B_{i}$. Let $T$ be the set of points $D_{i} \cap B_{i}$ for $i=1,2,3$; if $\#\left(T \cap D_{i}\right)<\operatorname{deg} D_{i}^{2}$, we add to $T$ smooth points of $D_{i}$ so that $\# T=\operatorname{deg} D_{1}^{2}+\operatorname{deg} D_{2}^{2}+\operatorname{deg} D_{3}^{2}$.

Let $X$ be the blowup $\mathbb{P}^{2}$ along $T$, and denote by $\widetilde{D}_{i}$ the strict transform of $D_{i}$ and by $\widetilde{H}$ the strict transform of $H$. Let $\Delta$ be the $\mathbb{Q}$-divisor defined as

$$
\Delta=\sum_{i=1}^{3}\left(1-\frac{1}{m_{i}}\right) \widetilde{D}_{i}+\left(1-\frac{1}{m_{4}}\right) \widetilde{H} .
$$


Then there exists a positive integer $m>0$ such that if $m_{i}>m$,

1. $(X, \Delta)$ is pseudo-algebraically hyperbolic and

2. if $\kappa=\mathbb{C}$, every orbifold entire curve $f: \mathbb{C} \rightarrow(X, \Delta)$ is degenerate.

Proof. Let $d_{i}=\operatorname{deg} D_{i}$. By definition, we have the following intersection numbers in $X: \widetilde{D}_{i} \cdot \widetilde{D}_{j}=d_{i} d_{j}$, $\widetilde{D}_{i}^{2}=0$ and $\widetilde{D}_{i} \cdot \widetilde{H}=d_{i}$. The result will follow from an application of Theorem 5.5. Let $c:=4 d_{1} d_{2} d_{3}$ and define $p_{i}:=c / d_{i}$ for $i=1,2,3$ and $p_{4}:=3 c / 4=3 d_{1} d_{2} d_{3}$. Using the integers $p_{1}, p_{2}, p_{3}$ and $p_{4}$, we define

$$
D_{p}=p_{1} \widetilde{D}_{1}+p_{2} \widetilde{D}_{2}+p_{3} \widetilde{D}_{3}+p_{4} \widetilde{H}
$$

Then it is immediate to verify that $D_{p} \cdot \widetilde{H}>0$ and $D_{p} \cdot E>0$, where $E$ is any exceptional divisor. Now let $C$ be a nonexceptional curve in $X$. Then we can compute

$$
D_{p} \cdot C=\left(\sum_{i=1}^{3} p_{i} d_{i}+p_{4}\right) H \cdot \pi_{*} C-\sum_{i=1}^{3} p_{i} \sum_{Q \in D_{i} \cap T} \operatorname{mult}_{Q}\left(\pi_{*} C\right) .
$$

Then, denoting $c=\operatorname{deg} \pi_{*} C$, Bézout's theorem implies that $\sum_{Q \in D_{i} \cap T}$ mult $_{Q}\left(\pi_{*} C\right) \leq c d_{i}$, and therefore we can rewrite equation (6.1) as

$$
D_{p} \cdot C \geq c\left(\sum_{i=1}^{3} p_{i} d_{i}+p_{4}\right)-\sum_{i=1}^{3} p_{i}\left(c d_{i}\right)=c p_{4}>0 .
$$

Finally, since $D_{p}^{2}>0$, by the Nakai-Moishezon criterion [Laz04, Theorem 1.2.23] the divisor $D_{p}$ is ample.

Let $i=1,2,3$ and let $\xi_{i}$ be the smallest solution of

$$
\widetilde{D}_{i}^{2} x^{2}-2\left(D_{p} \cdot \widetilde{D}_{i}\right) x+D_{p}^{2}=0
$$

Using the fact that $\widetilde{D}_{i}^{2}=0$ for $i=1,2,3$, this gives

$$
\xi_{i}=\frac{D_{p}^{2}}{2\left(D_{p} \cdot D_{i}\right)}
$$

Using this expression, to apply Theorems 5.5 and 5.11 we have to verify that the following inequality holds true:

$$
2 D_{p}^{2} \cdot \frac{D_{p}^{2}}{2\left(D_{p} \cdot \widetilde{D}_{i}\right)}>\left(D_{p} \cdot \widetilde{D}_{i}\right) \cdot \frac{D_{p}^{4}}{4\left(D_{p} \cdot \widetilde{D}_{i}\right)^{2}}+3 D_{p}^{2} \cdot p_{i} .
$$

This simplifies to $D_{p}^{2}>4 p_{i}\left(D_{p} \cdot \widetilde{D}_{i}\right)$. Using the definitions of $D_{p}$ and $p_{i}$, we can compute $D_{p}^{2}=177 d^{2}$, where $d=d_{1} d_{2} d_{3}$, and $D_{p} \cdot \widetilde{D}_{i} / d_{i}=11 d$. In particular, $4 p_{i}\left(D_{p} \cdot \widetilde{D}_{i}\right)=176 d^{2}$ so that formula (6.3) reads $177 d^{2}>176 d^{2}$ and therefore is verified in the case in which $i=1,2,3$.

To conclude, we need to verify that the same condition holds for $i=4$. In this case, using the fact that $D_{p} \cdot \widetilde{H}=15 d$, equation (6.2) becomes

$$
x^{2}-30 d x+177 d^{2}=0 .
$$

A direct computation shows that the smallest solution of the equation is $\xi=d(15-4 \sqrt{3})$. Then formula (6.3) becomes

$$
2 \cdot 177 \cdot(15-4 \sqrt{3})>15 \cdot(15-4 \sqrt{3})^{2}+9 \cdot 177,
$$


which is a true statement, thus concluding the verification of the hypotheses of Theorems 5.5 and 5.11, which imply the desired conclusion.

Remark 6.3. We note that the orbifold $(X, \Delta)$ will be of general type as soon as the $m_{i}$ s are big enough. Moreover, in the case in which all the multiplicities $m_{i}=\infty$, we recover the analogue of [CZ10, Proposition 2] in the function-field and Nevanlinna case.

\subsection{Weakly special threefolds}

In order to prove degeneracy results for the threefolds $X_{m}$ constructed in Section 4, we will use Proposition 6.2 in a special case that guarantees that the quasi-projective surface $S$ is not of $\log$ general type but the orbifold $(S, \Delta)$ is of orbifold general type.

Let $D:=D_{1}+D_{2}+D_{3}+D_{4}$ be a simple normal crossing divisor on $\mathbb{P}^{2}$, where $D_{1}$ is a curve of degree $d$ and $D_{2}, D_{3}, D_{4}$ are lines. Let $F:=F_{1}+F_{2}+F_{3}$ be a simple normal crossing divisor on $\mathbb{P}^{2}$, where $F_{1}$ is a curve of degree $d$ and $F_{2}, F_{3}$ are lines and such that $F+D$ is a simple normal crossing divisor (so that $\left(D_{i}, F_{i}\right)$ for $i=1,2,3$ and $D_{4}$ are in general position in the sense of Definition 6.1). Define $T_{i}:=D_{i} \cap F_{i}$, $1 \leq i \leq 3$, and let $T=T_{1} \cup T_{2} \cup T_{3}$. Let $\pi: \bar{S} \rightarrow \mathbb{P}^{2}$ be the blowup of $\mathbb{P}^{2}$ in $T$ and $\widetilde{D}_{i}=\pi_{*}^{-1} D_{i}$ be the strict transform of $D_{i}$ for $i=1, \ldots, 4$. In order to construct the fibration $g$ of Example 4.4, we consider the quasi-projective surface $S$ given by $\bar{S} \backslash \widetilde{D}_{2} \cup \widetilde{D}_{3} \cup \widetilde{D}_{4}$ and the fibration $g: S \rightarrow \mathbb{P}^{1}$ to be the fibration induced by $D_{1}$ and $F_{1}$. Note that for any integer $m \geq 2$, the divisor $K_{\mathbb{P}^{2}}+(1-1 / m) D_{1}+D_{2}+D_{3}+D_{4}$ is big, which implies in particular that the orbifold surface $\left(S,(1-1 / m) \widetilde{D}_{1}\right)$ is an orbifold of general type. Moreover, $S \backslash \widetilde{D}_{1}$ is simply connected (by the same argument as in Example 4.4).

Remark 6.4. The quasi-projective surface $\bar{S} \backslash \widetilde{D}_{1} \cup \widetilde{D}_{2} \cup \widetilde{D}_{3} \cup \widetilde{D}_{4}$ appears in [CZ10, Theorem 3] to produce an example of a simply connected quasi-projective surface with a non-Zariski dense set of integral points. The idea of our construction is to consider instead the orbifold surface $\left(\bar{S},(1-1 / m) \widetilde{D}_{1}+\widetilde{D}_{2}+\widetilde{D}_{3}+\widetilde{D}_{4}\right)$ and prove the degeneracy of orbifold entire curves. Unfortunately, it seems that the arithmetic orbifold analogue is out of reach with present methods.

Given $f$ as in Example 4.3, we can construct the smooth threefold $X_{m}$ as in Theorem 4.2: it is a weakly special threefold that is not special. We denote by $\pi: X_{m} \rightarrow S$ the elliptic fibration induced. Note that by construction, the orbifold base (see Definition 2.4) of $\pi$ coincides with $\Delta_{\pi}=(1-1 / m) \widetilde{D}_{1}$. Then the following theorem proves the degeneracy-result analogues of Conjecture 10 and the analytic part of Conjecture 1 for the quasi-projective threefold $X_{m}$ :

Theorem 6.5. There exists $m_{0}$ such that for all $m \geq m_{0}$, the following holds:

1. there exists a positive constant A such that for every morphism $\varphi: \mathcal{C} \rightarrow \bar{X}_{m}$ such that $\pi(\varphi(\mathcal{C}))$ is not contained in the exceptional set of Proposition 6.2, the following holds:

$$
\operatorname{deg} \pi(\varphi(\mathcal{C})) \leq A\left(2 g(\mathcal{C})-2+N_{\pi \circ \varphi}^{[1]}\left(\widetilde{D}_{2}+\widetilde{D}_{3}+\widetilde{D}_{4}\right)\right)
$$

2. every entire curve $f: \mathbb{C} \rightarrow X_{m}$ is algebraically degenerate.

In particular, the threefolds $X_{m}$ give counterexamples to (the logarithmic analogue of) Conjecture 12.

Proof. We first prove the statement in the function-field case. Let $\varphi$ be a morphism as before and consider $\pi \circ \varphi: \mathcal{C} \rightarrow \bar{S}$. By construction, this induces an orbifold morphism

$$
\left(\mathcal{C}, \varphi^{-1}\left(\widetilde{D}_{2}+\widetilde{D}_{3}+\widetilde{D}_{4}\right)\right) \rightarrow\left(\bar{S},\left(1-\frac{1}{m}\right) \widetilde{D}_{1}+\widetilde{D}_{2}+\widetilde{D}_{3}+\widetilde{D}_{4}\right)
$$

Then the conclusion follows from Proposition 6.2. Similarly, in the Nevanlinna case one considers an entire curve $f: \mathbb{C} \rightarrow X_{m}$ : the composition $\pi \circ f$ is an orbifold entire curve to $\left(\bar{S},(1-1 / m) \widetilde{D}_{1}+\widetilde{D}_{2}+\right.$ $\left.\widetilde{D}_{3}+\widetilde{D}_{4}\right)$. Then the conclusion follows again from Proposition 6.2. 


\section{The Ru-Vojta method}

The purpose of this section is to obtain a truncated version of the Ru-Vojta Theorem [RV20, General Theorem (Analytic Part)], as well as its analogue in the function-field setting.

\subsection{Nevanlinna theory}

We first recall some definitions in Nevanlinna theory. Let $D$ be an effective Cartier divisor on a complex variety $X$. Let $s=1_{D}$ be a canonical section of $\mathcal{O}(D)$ - that is, a global section for which $(s)=D-$ and fix a smooth metric $|\cdot|$ on $\mathcal{O}(D)$. The associated Weil function $\lambda_{D}: X(\mathbb{C}) \backslash \operatorname{Supp} D \rightarrow \mathbb{R}$ is given by

$$
\lambda_{D}(x):=-\log |s(x)|
$$

It is linear in $D$ (over a suitable domain), so by linearity and continuity it can be defined for a general Cartier divisor $D$ on $X$.

Let $f: \mathbb{C} \rightarrow X$ be a holomorphic map whose image is not contained in the support of divisor $D$ on $X$. The proximity function of $f$ with respect to $D$ is defined by

$$
m_{f}(D, r)=\int_{0}^{2 \pi} \lambda_{D}\left(f\left(r e^{i \theta}\right)\right) \frac{d \theta}{2 \pi} .
$$

Let $n_{f}(D, t)$ (resp., $\left.n_{f}^{(Q)}(D, t)\right)$ be the number of zeros of $\rho \circ f$ inside $\{|z|<t\}$, counting multiplicity (resp., ignoring multiplicity larger than $Q \in \mathbb{N}$ ) with $\rho$ a local equation of $D$. The counting function and the truncated counting function of $f$ of order $Q$ at $\infty$ are defined, respectively, by

$$
N_{f}(D, r)=\int_{1}^{r} \frac{n_{f}(D, t)}{t} d t \quad \text { and } \quad N_{f}^{(Q)}(D, r)=\int_{1}^{r} \frac{n_{f}^{Q}(D, t)}{t} d t .
$$

The height function relative to $D$ is defined, up to $O(1)$, as

$$
T_{D, f}(r)=m_{f}(D, r)+N_{f}(D, r) .
$$

The following is a truncated version of the analytic part of [RV20, General Theorem]:

Theorem 7.1. Let $X$ be a complex projective variety of dimension $n$, let $D_{1}, \ldots, D_{q}$ be effective Cartier divisors intersecting properly on $X$ and let $\mathcal{L}$ be a big line sheaf. For each $\varepsilon>0$, there exists a positive integer $Q$ such that for any algebraically nondegenerate holomorphic map $f: \mathbb{C} \rightarrow X$, the inequality

$$
\sum_{j=1}^{q} \beta\left(\mathcal{L}, D_{j}\right) T_{D_{j}, f}(r)-(1+\varepsilon) T_{\mathcal{L}, f}(r) \leq_{\operatorname{exc}} \sum_{j=1}^{q} \beta\left(\mathcal{L}, D_{j}\right) N_{f}^{(Q)}\left(D_{j}, r\right)
$$

holds, where $\leq_{\mathrm{exc}}$ means that the inequality holds for all $r \in \mathbb{R}^{+}$except a set of finite Lebesgue measure.

Remark 7.2. When $X$ is a surface, the condition that $D_{1}, \ldots, D_{q}$ intersect properly on $X$ can be relaxed to a general-position assumption, following the same strategy as in [HR18, Main Theorem].

We recall the following theorem from [GW19], which is a modification of [HR18, Theorem 3.2], by applying the general form of the second main theorem with a Wronskian term proved by Vojta in [Voj97, Theorem 1] and Ru in [Ru97, Theorem 2.3].

Theorem 7.3. Let $X$ be a complex projective variety, $D$ be a Cartier divisor on $X, V$ be a nonzero linear subspace of $H^{0}(X, \mathcal{O}(D))$ and $s_{1}, \ldots, s_{q}$ be nonzero elements of $V$, and for each $j=1, \ldots, q$, let $D_{j}$ be the Cartier divisor $\left(s_{j}\right)$. 
Let $\Phi=\left(\phi_{1}, \ldots, \phi_{d}\right): X \rightarrow \mathbb{P}^{d-1}$ be the rational map associated to the linear system $V, f: \mathbb{C} \rightarrow X$ be a holomorphic map with Zariski dense image and $\Psi=\left(\psi_{1}, \ldots, \psi_{d}\right): \mathbb{C} \rightarrow \mathbb{P}^{d-1}$ be a reduced form of $\Phi \circ f-$ that is, $\Psi=\Phi \circ f$ and $\psi_{1}, \ldots, \psi_{d}$ are entire functions without common zeros. Denote by $W(\Psi)$ the Wronskian of $\psi_{1}, \ldots, \psi_{d}$. Then for any $\varepsilon>0$,

$$
\int_{0}^{2 \pi} \max _{J} \sum_{j \in J} \lambda_{D_{j}}\left(f\left(r e^{i \theta}\right)\right) \frac{d \theta}{2 \pi}+N_{W(\Psi)}(0, r)+\operatorname{dim} V \cdot N_{h}(0, r) \leq_{\operatorname{exc}}(\operatorname{dim} V+\varepsilon) T_{D, f}(r)
$$

where $J$ ranges over all subsets of $\{1, \ldots, q\}$ such that the sections $\left(s_{j}\right)_{j \in J}$ are linearly independent and $h$ is an an entire function such that $h \psi_{i}=\phi_{i}(f)$ for each $1 \leq i \leq d-$ that is, $h$ is a greatest common divisor of $\phi_{i}(f), 1 \leq i \leq d$.

Proof. Most of the proof is identical to the proof of [HR18, Theorem 3.2], so we will omit some details and indicate the required adjustments.

We may assume that $d>1$. Let $X^{\prime}$ be the closure of the graph of $\Phi$, and let $p: X^{\prime} \rightarrow X$ and $\phi: X^{\prime} \rightarrow \mathbb{P}^{d-1}$ be the projection morphisms. Then there is an effective Cartier divisor $B$ on $X^{\prime}$ such that for each nonzero $s \in V$, there is a hyperplane $H$ in $\mathbb{P}^{d-1}$ such that $p^{*}(s)-B=\phi^{*} H$. Let $\tilde{f}: \mathbb{C} \rightarrow X^{\prime}$ be the lifting of $f$. Then $\phi \circ \tilde{f}=\left(\psi_{1}, \ldots, \psi_{d}\right): \mathbb{C} \rightarrow \mathbb{P}^{d-1}$ is a reduced presentation of $\Phi \circ f$. Moreover, let $h$ be an entire function such that $h \psi_{i}=\phi_{i}(f)$ for each $1 \leq i \leq d-$ that is, $h$ is a greatest common divisor of $\phi_{i}(f), 1 \leq i \leq d$. Then

$$
N_{\tilde{f}}(B, r) \geq N_{h}(0, r)+O(1)
$$

For each $j=1, \ldots, q$, let $H_{j}$ be the hyperplane in $\mathbb{P}^{d-1}$ for which

$$
p^{*} D_{j}-B=p^{*}\left(s_{j}\right)-B=\phi^{*} H_{j}
$$

Choose a Weil function $\lambda_{B}$ for $B$. Then we have

$$
\lambda_{p^{*} D_{j}}=\lambda_{\phi^{*} H_{j}}+\lambda_{B}+O(1)
$$

By the functoriality of Weil functions,

$$
\lambda_{p^{*} D_{j}}(\tilde{f}(z))=\lambda_{D_{j}}(f(z)) \quad \text { and } \quad \lambda_{\phi^{*} H_{j}}\left(\tilde{f}\left(r e^{i \theta}\right)\right)=\lambda_{H_{j}}\left(\phi\left(\tilde{f}\left(r e^{i \theta}\right)\right)\right) .
$$

By the general form of the second main theorem with a Wronskian term in [Voj97, Theorem 1] and [Ru97, Theorem 2.3], we have

$$
\int_{0}^{2 \pi} \max _{J} \sum_{j \in J} \lambda_{H_{j}}\left(\phi(\tilde{f})\left(r e^{i \theta}\right)\right) \frac{d \theta}{2 \pi}+N_{W(\phi(\tilde{f}))}(0, r) \leq_{\operatorname{exc}}(d+\varepsilon) T_{\phi(\tilde{f})}(r) .
$$

From equation (7.3), we have

$$
T_{\phi(\tilde{f})}(r)=T_{D, f}-T_{B, \tilde{f}}
$$

Since each set $J$ has atmost $\operatorname{dim} V$ elements and $B$ is effective, it follows from formula (7.2) that

$$
(\# J)\left(m_{\tilde{f}}(B, r)+N_{h}(0, r)\right) \leq(\# J)\left(m_{\tilde{f}}(B, r)+N_{\tilde{f}}(B, r)\right) \leq d T_{B, \tilde{f}}+O(1) .
$$


Hence,

$$
\begin{aligned}
& \left.\int_{0}^{2 \pi}\left(\max _{J} \sum_{j \in J} \lambda_{H_{j}}\left(\phi(\tilde{f})\left(r e^{i \theta}\right)\right)+\lambda_{B}\left(\tilde{f}\left(r e^{i \theta}\right)\right)\right) \frac{d \theta}{2 \pi}+d N_{h}(0, r)\right)+N_{W(\phi(\tilde{f}))}(0, r) \\
& \leq_{\mathrm{exc}}(d+\varepsilon) T_{\phi(\tilde{f})}(r)+d T_{B, \tilde{f}}+O(1) \\
& \leq_{\mathrm{exc}}(d+\varepsilon) T_{D, f}+O(1) .
\end{aligned}
$$

Proof of Theorem 7.1. We will follow the proof of the analytic part of [RV20, General Theorem] closely, and only indicate the necessary modification. Let $\epsilon>0$ be given. We want to show that

$$
\sum_{j=1}^{q} \beta\left(\mathcal{L}, D_{j}\right)\left(T_{D_{j}, f}(r)-N_{f}^{(Q)}\left(D_{j}, r\right)\right) \leq_{\text {exc }}(1+\varepsilon) T_{\mathcal{L}, f}(r) .
$$

Since the quantities $\left(T_{D_{j}, f}(r)-N_{f}^{(Q)}\left(D_{j}, r\right)\right) / T_{\mathcal{L}, f}(r)$ are bounded when their respective denominators are sufficiently large, it suffices to prove formula (7.4) with a slightly smaller $\epsilon>0$ and with $\beta\left(\mathcal{L}, D_{i}\right)$ replaced by slightly smaller $\beta_{i} \in \mathbb{Q}$ for all $i$. It is also clear that we may assume that $\beta_{i} \neq 0$ for each $i$.

Choose positive integers $N$ and $b$ such that

$$
\left(1+\frac{n}{b}\right) \max _{1 \leq i \leq q} \frac{\beta_{i} N h^{0}\left(X, \mathcal{L}^{N}\right)}{\sum_{m \geq 1} h^{0}\left(X, \mathcal{L}^{N}\left(-m D_{i}\right)\right)}<1+\epsilon .
$$

Let

$$
\Sigma=\left\{\sigma \subseteq\{1, \ldots, q\} \mid \bigcap_{j \in \sigma} \operatorname{Supp} D_{j} \neq \emptyset\right\}
$$

For $\sigma \in \Sigma$, let

$$
\Delta_{\sigma}=\left\{\mathbf{a}=\left(a_{i}\right) \in \prod_{i \in \sigma} \beta_{i}^{-1} \mathbb{N} \mid \sum_{i \in \sigma} \beta_{i} a_{i}=b\right\} .
$$

For $\mathbf{a} \in \triangle_{\sigma}$ as before, we construct a filtration of $H^{0}\left(X, \mathcal{L}^{N}\right)$ as follows: for $x \in \mathbb{R}^{+}$, we define the ideal $\mathcal{J}_{\mathbf{a}}(x)$ of $\mathcal{O}_{X}$ by

$$
\mathcal{J}_{\mathbf{a}}(x)=\sum_{\mathbf{b}} \mathcal{O}_{X}\left(-\sum_{i \in \sigma} b_{i} D_{i}\right)
$$

where the sum is taken for all $\mathbf{b} \in \mathbb{N}^{\# \sigma}$ with $\sum_{i \in \sigma} a_{i} b_{i} \geq b x$. Let

$$
\mathcal{F}(\sigma ; \mathbf{a})_{x}=H^{0}\left(X, \mathcal{L}^{N} \otimes \mathcal{J}_{\mathbf{a}}(x)\right),
$$

which we regard as a subspace of $H^{0}\left(X, \mathcal{L}^{N}\right)$. We note that there are only finitely many ordered pairs $(\sigma, \mathbf{a})$ with $\sigma \in \Sigma$ and $\mathbf{a} \in \Delta_{\sigma}$. Let $\mathcal{B}_{\sigma ; \mathbf{a}}$ be a basis of $H^{0}\left(X, \mathcal{L}^{N}\right)$ adapted to the filtration $\left\{\mathcal{F}(\sigma ; \mathbf{a})_{x}\right\}_{x \in \mathbb{R}^{+}}$.

For a basis $\mathcal{B}$ of $H^{0}\left(X, \mathcal{L}^{N}\right)$, denote by $(\mathcal{B})$ the sum of the divisors $(s)$ for all $s \in \mathcal{B}$. We now state the following main lemma in [RV20]: 
Lemma 7.4 ([RV20, Lemma 6.8]). With the previous notation, we have

$$
\bigvee_{\substack{\sigma \in \Sigma \\ \mathbf{a} \in \Delta_{\sigma}}}\left(\mathcal{B}_{\sigma ; \mathbf{a}}\right) \geq \frac{b}{b+n}\left(\min _{1 \leq i \leq q} \frac{\sum_{m=1}^{\infty} h^{0}\left(X, \mathcal{L}^{N}\left(-m D_{i}\right)\right)}{\beta_{i}}\right) \sum_{i=1}^{q} \beta_{i} D_{i}
$$

The notation $\bigvee$ is referred to the least upper bound with respect to the partial order on the (Cartier) divisor group of $X$ by the relation $D_{1} \leq D_{2}$ if $D_{2}-D_{1}$ is effective. Write

$$
\bigcup_{\sigma ; \mathbf{a}} \mathcal{B}_{\sigma ; \mathbf{a}}=\mathcal{B}_{1} \cup \cdots \cup \mathcal{B}_{T_{1}}=\left\{s_{1}, \ldots, s_{T_{2}}\right\}
$$

For each $i=1, \ldots, T_{1}$, let $J_{i} \subseteq\left\{1, \ldots, T_{2}\right\}$ be the subset such that $\mathcal{B}_{i}=\left\{s_{j}: j \in J_{i}\right\}$. Choose Weil functions $\lambda_{\mathcal{B}_{i}}\left(i=1, \ldots, T_{1}\right)$ and $\lambda_{s_{j}}\left(j=1, \ldots, T_{2}\right)$ for the divisors $\left(\mathcal{B}_{i}\right)$ and $\left(s_{j}\right)$, respectively. Then, by formula (7.6) for $x \in X$,

$$
\begin{aligned}
& \frac{b}{b+n}\left(\min _{1 \leq i \leq q} \sum_{m \geq 1} \frac{h^{0}\left(\mathcal{L}^{N}\left(-m D_{i}\right)\right)}{\beta_{i}}\right) \sum_{i=1}^{q} \beta_{i} \lambda_{D_{i}}(x) \\
& \quad \leq \max _{1 \leq i \leq T_{1}} \lambda_{\mathcal{B}_{i}}(x)+O(1)=\max _{1 \leq i \leq T_{1}} \sum_{j \in J_{i}} \lambda_{s_{j}}(x)+O(1) .
\end{aligned}
$$

Let $M=h^{0}\left(X, \mathcal{L}^{N}\right)$, let the set $\left\{\phi_{1}, \ldots, \phi_{M}\right\}$ be a basis of the vector space $H^{0}\left(X, \mathcal{L}^{N}\right)$ and let $\Phi=\left[\phi_{1}, \ldots, \phi_{M}\right]: X \rightarrow \mathbb{P}^{M-1}(\mathbb{C})$ be the corresponding (rational) map. Let $f: \mathbb{C} \rightarrow X$ be an algebraically nondegenerate holomorphic map. Let $h$ be a greatest common divisor of $\phi_{1}(f), \ldots, \phi_{M}(f)$ - that is, $h$ is an entire function such that $\psi_{1}:=h^{-1} \phi_{1}(f), \ldots, \psi_{M}:=h^{-1} \phi_{M}(f)$ have no common zeros. Then $\Psi=\left(\psi_{1}, \ldots, \psi_{M}\right)$ is a reduced form of $\Phi \circ f$.

By Theorem 7.3,

$$
\int_{0}^{2 \pi} \max _{J} \sum_{j \in J} \lambda_{s_{j}}\left(f\left(r e^{i \theta}\right)\right) \frac{d \theta}{2 \pi} \leq_{\mathrm{exc}}(M+\epsilon) T_{f, \mathscr{L}^{N}}(r)-N_{W(\Psi)}(0, r)-M N_{h}(0, r) ;
$$

here the maximum is taken over all subsets $J$ of $\left\{1, \ldots, T_{2}\right\}$ for which the sections $s_{j}, j \in J$, are linearly independent. Combining formulas (7.7) and (7.8), we have

$$
\begin{aligned}
& \sum_{i=1}^{q} \beta_{i} m_{f}\left(D_{i}, r\right) \\
& \leq_{\operatorname{exc}}\left(1+\frac{n}{b}\right) \max _{1 \leq i \leq q} \frac{\beta_{i}}{\sum_{m \geq 1} h^{0}\left(\mathscr{L}^{N}\left(-m D_{i}\right)\right)}\left((M+\epsilon) T_{f, \mathscr{L}^{N}}(x)-N_{W(\Psi)}(0, r)-M N_{h}(0, r)\right) .
\end{aligned}
$$

Using formula (7.5) and the fact that $T_{f, \mathscr{L}^{N}}(r)=N T_{f, \mathscr{L}}(r)$, we have

$$
\sum_{i=1}^{q} \beta_{i} m_{f}\left(D_{i}, r\right) \leq(1+2 \epsilon) T_{f, \mathscr{L}}(x)-C\left(N_{W(\Psi)}(0, r)+M N_{h}(0, r)\right)
$$

where $C=(1+\epsilon)(M N)^{-1}$. Applying equation (7.1) to this inequality, we have

$$
\sum_{\substack{j=1 \\ \text { ind }}}^{q} \beta_{j} T_{D_{j}, f}(r)-(1+2 \varepsilon) T_{\mathscr{L}, f}(r) \leq_{\text {exc }} \sum_{\substack{j=1 \\ \text { f }}}^{q} \beta_{j} N_{f}\left(D_{j}, r\right)-C\left(N_{W(\Psi)}(0, r)+M N_{h}(0, r)\right) .
$$


To finish the proof, we will show that there exists a large integer $Q$ (to be determined later) such that

$$
\sum_{j=1}^{q} \beta_{j} N_{f}\left(D_{j}, r\right)-C\left(N_{W(\Psi)}(0, r)+M N_{h}(0, r)\right) \leq \sum_{j=1}^{q} \beta_{j} N_{f}^{(Q)}\left(D_{j}, r\right) .
$$

For $z_{0} \in \mathbb{C}$, let $\rho_{j}$ be a local defining function of $D_{j}$ around one of its open neighborhoods $U$. To show formula (7.9)), it suffices to deduce the inequality

$$
\sum_{j=1}^{q} \beta_{j} v_{z_{0}}^{+}\left(\rho_{j} \circ f\right)-C \cdot\left(v_{z_{0}}^{+}(W(\Psi))+M v_{z_{0}}(h)\right) \leq \sum_{j=1}^{q} \beta_{j} \min \left\{Q, v_{z_{0}}^{+}\left(\rho_{j} \circ f\right)\right\}
$$

for each $z_{0} \in \mathbb{C}$.

This inequality holds trivially if $v_{z_{0}}^{+}\left(\rho_{j} \circ f\right) \leq Q$ for each $1 \leq j \leq q$. Therefore, we assume that $v_{z_{0}}^{+}\left(\rho_{j} \circ f\right) \geq Q$ for some $1 \leq j \leq q$. By Lemma 7.4, there exists a set of basis $s_{1}, \ldots, s_{M}$ of $H^{0}\left(X, \mathscr{L}^{N}\right)$ with the following property:

$$
\begin{aligned}
\sum_{i=1}^{M}\left(s_{i}\right) & \geq \frac{b}{b+n}\left(\min _{1 \leq i \leq q} \sum_{m=1}^{\infty} \frac{h^{0}\left(X, \mathscr{L}^{N}\left(-m D_{i}\right)\right)}{\beta_{i}}\right) \sum_{i=1}^{q} \beta_{i} D_{i} \\
& \geq \frac{M N}{1+\epsilon} \sum_{i=1}^{q} \beta_{i} D_{i}=\frac{1}{C} \sum_{i=1}^{q} \beta_{i} D_{i},
\end{aligned}
$$

where the last inequality is due to formula (7.5). Since

$$
v_{z_{0}}^{+}\left(\left.s_{i}\right|_{U} \circ f\right) \geq \sum_{j=1}^{q} \operatorname{ord}_{D_{j}} s_{i} \cdot v_{z_{0}}^{+}\left(\rho_{j} \circ f\right),
$$

we can derive from formula (7.10) that

$$
\sum_{i=1}^{M} v_{z_{0}}^{+}\left(\left.s_{i}\right|_{U} \circ f\right) \geq \frac{1}{C} \sum_{j=1}^{q} \beta_{j} v_{z_{0}}^{+}\left(\rho_{j} \circ f\right) .
$$

On the other hand, since $\left\{\phi_{1}, \ldots, \phi_{M}\right\}$ is a basis of the vector space $H^{0}\left(X, \mathscr{L}^{N}\right)$, each $s_{j}$ is a $\mathbb{C}$-linear combination of the $\phi_{i} \mathrm{~s}$, and hence each $h^{-1} s_{j}(f)$ is a linear combination of the $\psi_{i}$ s. From the basic properties of Wronskians, we have

$$
v_{z_{0}}^{+}(W(\Psi)) \geq \sum_{j=1}^{M} v_{z_{0}}^{+}\left(\left.s_{j}\right|_{U} \circ f\right)-M v_{z_{0}}(h)-\frac{1}{2} M(M-1) .
$$

Combining formula (7.11)) and (7.12) and the assumption that $v_{z_{0}}^{+}\left(\rho_{j} \circ f\right) \geq Q$ for some $1 \leq j \leq q$, we obtain

$$
\begin{aligned}
\sum_{j=1}^{q} \beta_{j} v_{z_{0}}^{+}\left(\rho_{j} \circ f\right)-C \cdot\left(v_{z_{0}}^{+}(W(\Phi \circ f))+M v_{z_{0}}(h)\right) & \leq \frac{1}{2} C M(M-1) \\
& \leq \sum_{j=1}^{q} \beta_{j} \min \left\{Q, v_{z_{0}}^{+}\left(\rho_{j} \circ f\right)\right\},
\end{aligned}
$$

where $Q$ is chosen to be $\frac{C M(M-1)}{2 \min _{1 \leq j \leq q}\left\{\beta_{j}\right\}}$. (Note that we have assumed that $\beta_{j} \neq 0$ for each $j$.) This completes our proof. 


\subsection{Function fields}

In this section we let $\kappa$ be an algebraically closed field of characteristic $1, \mathcal{C}$ be a smooth projective curve over $\kappa$ of genus $g(\mathcal{C})$ and $K=\kappa(\mathcal{C})$ be the function field of $\mathcal{C}$. For each point $\mathfrak{p} \in \mathcal{C}$, we may choose a uniformiser $t_{\mathfrak{p}}$ to define a normalised order function $v_{\mathfrak{p}}:=\operatorname{ord}_{\mathfrak{p}}: K \rightarrow \mathbb{Z} \cup\{\infty\}$ at $\mathfrak{p}$. For a nonzero element $f \in K$, the height $h(f)$ counts its number of poles with multiplicities - that is,

$$
h(f):=\sum_{\mathfrak{p} \in \mathcal{C}} \max \left\{0,-v_{\mathfrak{p}}(f)\right\} .
$$

Set $f_{0}, \ldots, f_{m} \in K$ not all zeros. Then $\mathbf{f}=\left[f_{0}: \cdots: f_{m}\right] \in \mathbb{P}^{m}(K)$ can be viewed as a morphism from $\mathcal{C}$ to $\mathbb{P}^{m}(\kappa)$. The height of this morphism (or of the corresponding point in $\mathbb{P}^{m}(K)$ ) is defined by

$$
h(\mathbf{f})=h\left(f_{0}, \ldots, f_{m}\right):=\sum_{\mathfrak{p} \in \mathcal{C}}-\min \left\{v_{\mathfrak{p}}\left(f_{0}\right), \ldots, v_{\mathfrak{p}}\left(f_{m}\right)\right\} .
$$

Let $D$ be a Cartier divisor on a variety $X$ over $\kappa$. Similar to the number-field case, the classical theory of heights (see, for example, [HS00, Part B] or [BG06, Chapter 2]) associates to every Cartier divisor $D$ on $X$ a height function $h_{D}: X(K) \rightarrow \mathbb{R}$ and a Weil function (local height function) $\lambda_{D, \mathfrak{p}}: X(K) \backslash \operatorname{Supp} D \rightarrow \mathbb{R}, \mathfrak{p} \in \mathcal{C}$, well defined up to a bounded function such that

$$
\sum_{\mathfrak{p} \in \mathcal{C}} \lambda_{D, \mathfrak{p}}(P)=h_{D}(P)+O(1)
$$

for all $P \in X(K) \backslash \operatorname{Supp} D$. Let $S$ be a finite set of points in $\mathcal{C}$. We denote by

$$
m_{D, S}(P)=\sum_{\mathfrak{p} \in S} \lambda_{D, \mathfrak{p}}(P) \quad \text { and } \quad N_{D, S}(P)=\sum_{\mathfrak{p} \notin S} \lambda_{D, \mathfrak{p}}(P)
$$

the proximity and counting functions as defined in [Voj87]. Let $a_{0} X_{0}+a_{1} X_{1}+\cdots+a_{m} X_{m}$ be a linear form with $a_{0}, \ldots, a_{m} \in \kappa$ whose vanishing determines a hyperplane $H$ in $\mathbb{P}^{m}$. Then for all $\mathfrak{p} \in \mathcal{C}$ and $P=\left[x_{0}: \cdots: x_{m}\right] \in \mathbb{P}^{m}(K)$, the Weil function at $\mathfrak{p}$ is given by

$$
\lambda_{H, \mathfrak{p}}(P)=v_{\mathfrak{p}}\left(a_{0} x_{0}+a_{1} x_{1}+\cdots+a_{m} x_{m}\right)-\min \left\{v_{\mathfrak{p}}\left(x_{0}\right), \ldots, v_{\mathfrak{p}}\left(x_{m}\right)\right\} .
$$

We recall the following version of the second main theorem for function fields from [Wan04]:

Theorem 7.5 ([Wan04, Theorem 1]). In the foregoing setting, let $H_{1}, \ldots, H_{q}$ be hyperplanes in $\mathbb{P}^{m}(K)$ defined by linear forms with coefficients in $\kappa$. If $P=\left[x_{0}: \cdots: x_{m}\right] \in \mathbb{P}^{m}(K)$ is linearly nondegenerate over $\kappa$, then

$$
\sum_{\mathfrak{p} \in S} \max _{j \in J} \lambda_{H_{j}, \mathfrak{p}}(P) \leq(m+1) h(P)+\frac{m(m+1)}{2}(2 g(\mathcal{C})-2+\# S),
$$

where the maximum is taken over all subsets $J$ of $\{1, \ldots, q\}$ such that the linear forms defining $H_{j}$, $j \in J$, are linearly independent.

We will use Theorem 7.5 to obtain a function-field analogue of Theorem 7.1 with an explicit dependence on the Euler characteristic of the curve $\mathcal{C}$.

Theorem 7.6. Let $X \subset \mathbb{P}^{m}$ be a projective variety over $\kappa$ of dimension $n, D_{1}, \ldots, D_{q}$ be effective Cartier divisors intersecting properly on $X$ and $\mathcal{L}$ be a big line sheaf. Then for any $\epsilon>0$, there exist constants $c_{1}$ and $c_{2}$, independent of the curve $\mathcal{C}$ and the set $S$, such that for any map $x=\left[x_{0}: \cdots: x_{m}\right]: \mathcal{C} \rightarrow X$, where $x_{i} \in K$, outside the augmented base locus of $\mathcal{L}$ either 


$$
\sum_{i=1}^{q} \beta_{\mathcal{L}, D_{i}} m_{D_{i}, S}(x) \leq(1+\epsilon) h_{\mathcal{L}}(x)+c_{1} \max \{1,2 g(\mathcal{C})-2+|S|\}
$$

or the image of $x$ is contained in a hypersurface (over $\kappa)$ in $\mathbb{P}^{m}$ of degree at most $c_{2}$.

Proof. The proof is similar to the first part of the proof of Theorem 7.1. We will follow its argument and notation and indicate only the modifications. Let $\epsilon>0$ be given. Since $\mathcal{L}$ is a big line sheaf, there is a constant $c$ such that $\sum_{i=1}^{q} h_{D_{i}}(x) \leq c h_{\mathcal{L}}(x)$ for all $x \in X(K)$ outside the augmented base locus $B$ of $\mathcal{L}$. This follows from [Voj11, Proposition 10.11]. By equation (7.13), together with the fact that $m_{D_{i}, S} \leq h_{D_{i}}+O(1)$, we can choose $\beta_{i} \in \mathbb{Q}$ for all $i$ such that

$$
\sum_{i=1}^{q}\left(\beta_{\mathcal{L}, D_{i}}-\beta_{i}\right) m_{D_{i}, S}(x) \leq \frac{\epsilon}{2} h_{\mathcal{L}}(x)
$$

for all $x \in X \backslash B(K)$. Therefore, we can assume that $\beta_{\mathcal{L}, D_{i}}=\beta_{i} \in \mathbb{Q}$ for all $i$. It is also clear that we may assume that $\beta_{i} \neq 0$ for each $i$. From now on we will assume that the point $x \in X(K)$ does not lie on $B$.

Choose positive integers $N$ and $b$ to satisfy formula (7.5). The same arguments as in the proof of Theorem 7.1 give

$$
\begin{aligned}
& \frac{b}{b+n}\left(\min _{1 \leq i \leq q} \sum_{m \geq 1} \frac{h^{0}\left(\mathcal{L}^{N}\left(-m D_{i}\right)\right)}{\beta_{i}}\right) \sum_{i=1}^{q} \beta_{i} \lambda_{D_{i}, \mathfrak{p}}(x) \\
& \quad \leq \max _{1 \leq i \leq T_{1}} \lambda_{\mathcal{B}_{i}, \mathfrak{p}}(x)+O(1)=\max _{1 \leq i \leq T_{1}} \sum_{j \in J_{i}} \lambda_{s_{j}, \mathfrak{p}}(x)+O(1) .
\end{aligned}
$$

Let $M=h^{0}\left(X, \mathcal{L}^{N}\right)$, the set $\left\{\phi_{1}, \ldots, \phi_{M}\right\}$ be a basis of the vector space $H^{0}\left(X, \mathcal{L}^{N}\right)$ and

$$
\Phi=\left[\phi_{1}, \ldots, \phi_{M}\right]: X \rightarrow \mathbb{P}^{M-1}(\kappa)
$$

be the corresponding rational map. By Theorem 7.5, either the map $\Phi \circ x$ is linearly degenerate - that is, $\phi_{1}(x), \ldots, \phi_{M}(x)$ are linearly dependent over $\kappa-$ or

$$
\sum_{\mathfrak{p} \in S} \max _{J} \sum_{j \in J} \lambda_{s_{j}, \mathfrak{p}}(x) \leq M h_{\mathcal{L}^{N}}(x)+\frac{M(M-1)}{2}(2 g-2+|S|),
$$

with the maximum here taken over all subsets $J$ of $\left\{1, \ldots, T_{2}\right\}$ for which the sections $s_{j}, j \in J$, are linearly independent. We first consider when $\phi_{1}, \ldots, \phi_{M}$ are linearly independent over $\kappa$. Combining formulas (7.14) and (7.16) gives

$$
\sum_{i=1}^{q} \beta_{i} m_{D_{i}, S}(x) \leq\left(1+\frac{n}{b}\right) \max _{1 \leq i \leq q} \frac{\beta_{i}}{\sum_{m \geq 1} h^{0}\left(\mathcal{L}^{N}\left(-m D_{i}\right)\right)} M h_{\mathcal{L}^{N}}(x)+c_{1}^{\prime}(2 g-2+|S|)+O(1),
$$

where $c_{1}^{\prime}=\frac{M(M-1)}{2}$. Using formula (7.5) and the fact that $h_{\mathcal{L}^{N}}(x)=N h_{\mathcal{L}}(x)$, we have

$$
\sum_{i=1}^{q} \beta_{i} m_{D_{i}, S}(x) \leq(1+\epsilon) h_{\mathcal{L}}(x)+c_{1}^{\prime}(2 g-2+|S|)+O(1),
$$

which implies the first case of the theorem.

To conclude, we note that if $\phi_{1}(x), \ldots, \phi_{M}(x)$ are linearly dependent over $\kappa$, there exist constants $a_{1}, \ldots, a_{M} \in \kappa$, not all zero, such that $a_{1} \phi_{1}(x)+\cdots+a_{M} \phi_{M}(x)=0$. Let $H$ be the hyperplane in $\mathbb{P}^{M-1}$ defined by $a_{1} z_{1}+\cdots+a_{M} z_{M}=0$; by assumption, $\Phi(x(\mathcal{C}))$ is contained in $H$. On the other hand, since 
$\phi_{1}, \ldots, \phi_{M}$ is a basis of $H^{0}\left(X, \mathcal{L}^{N}\right)$, it follows that $\Phi(X)$ is not contained in $H$, and hence $\Phi(x(\mathcal{C}))$ is contained in $\Phi(X) \cap H$, which is a hypersurface in $\Phi(X)$ whose degree is bounded independently of $\mathcal{C}$ and $x$, as wanted.

Corollary 7.7. In the previous setting, if $X$ has dimension 2 , then for any $\epsilon>0$, there exist constants $c_{1}$ and $c_{3}$, independent of the curve $\mathcal{C}$ and the set $S$, such that for any $K$-point $x=\left[x_{0}: \cdots: x_{m}\right]: \mathcal{C} \rightarrow X$, with $x_{i} \in K$, we have either $\operatorname{deg} x(\mathcal{C}) \leq c_{3}$ or

$$
\sum_{i=1}^{q} \beta\left(\mathcal{L}, D_{i}\right) m_{D_{i}, S}(x) \leq(1+\epsilon) h_{\mathcal{L}}(x)+c_{1} \max \{1,2 g(\mathcal{C})-2+|S|\} .
$$

Proof. Given Theorem 7.6, it is enough to observe that since $X$ has dimension 2, the hypersurface of degree bounded by $c_{2}$ intersects $X$ in a union of finitely many curves whose degree is bounded independently of the point $x$. Moreover, the augmented base locus of $\mathcal{L}$ has dimension at most 1 , and its one-dimensional locus is a union of finitely many curves which are also independent of $x$. Therefore, it suffices to define $c_{3}$ to the be the maximum of the degrees of all these finitely many curves.

Acknowledgments. We thank Laura Capuano, Pietro Corvaja, Lionel Darondeau, Carlo Gasbarri, Ariyan Javanpeykar, Stefan Kebekus and Min Ru for useful discussions. We thank Min Ru and Paul Vojta for providing us with an earlier copy of their paper. We are especially grateful to Frédéric Campana for all the discussions on this subject, and especially his explanations on the construction of weakly special but not special varieties. We thank the referees for their comments and suggestions, which greatly improved the paper.

This collaboration was initiated at the conference 'Topics on Nevanlinna Theory and Complex Hyperbolicities' at the Shanghai Center for Mathematical Sciences; we thank the organisers for making this possible. Part of this work was finalised during the visit of the first and second authors to the Freiburg Institute for Advanced Studies; they thank the Institute for providing an excellent working environment.

\section{Conflict of Interest: None.}

Financial support. The first author was supported by the European Union's Horizon 2020 research and innovation program under the Marie Skłodowska-Curie grant agreement No 754340, and by the ANR project 'FOLIAGE', ANR-16-CE40-0008. The second author was supported in part by funds from NSF grant DMS-1553459 and in part by Centro di Ricerca Matematica Ennio de Giorgi, and is a memeber of GNSAGA of INdAM. The third author was supported by Taiwan's MoST 108-2115-M-001001-MY2.

\section{References}

[ADT20] K. Ascher, K. DeVleming and A. Turchet, 'Hyperbolicity and uniformity of varieties of log general type', International Mathematics Research Notices, 2020, published online. https://doi.org/10.1093/imrn/rnaa186

[Aut11] P. Autissier, 'Sur la non-densité des points entiers', Duke Math. J. 158(1) (2011), 13-27.

[BG06] E. Bombieri and W. Gubler, Heights in Diophantine Geometry, New Mathematical Monographs, 4 ( Cambridge University Press, Cambridge, 2006).

[Bog79] F. A. Bogomolov, 'Holomorphic tensors and vector bundles on projective varieties', Math. USSR Izv. 13 (1979), 499-555.

[BRT19] F. Lo Bianco, E. Rousseau and F. Touzet, 'Symmetries of transversely projective foliations', Preprint, 2019, arXiv: 1901.05656.

[BT04] F. Bogomolov and Y. Tschinkel, 'Special elliptic fibrations', in The Fano Conference (University of Torino, Turin, Italy, 2004), 223-234.

[Cam04] F. Campana, 'Orbifolds, special varieties and classification theory', Ann. Inst. Fourier (Grenoble) 54(3) (2004), 499-630.

[Cam11] F. Campana, 'Orbifoldes géométriques spéciales et classification biméromorphe des variétés kählériennes compactes', J. Inst. Math. Jussieu 10(4) (2011), 809-934.

[Che04] X.Chen, 'On algebraic hyperbolicity of log varieties', Commun. Contemp. Math. 6(4) (2004), 513-559.

[CP07] F. Campana and M. Păun, 'Variétés faiblement spéciales à courbes entières dégénérées', Compos. Math. 143(1) (2007), 95-111.

[CT19] L. Capuano and A. Turchet, 'Lang-Vojta conjecture over function fields for surfaces dominating $G_{m}^{2}$ ', Preprint, 2019, arXiv: 1911.07562.

[CZ04] P. Corvaja and U. Zannier, 'On integral points on surfaces', Ann. of Math. (2) 160(2) (2004), 705-726. 
[CZ08] P. Corvaja and U. Zannier, 'Some cases of Vojta's Conjecture on integral points over function fields', J. Algebraic Geometry 17 (2008), 195-333.

[CZ10] P. Corvaja and U. Zannier, 'Integral points, divisibility between values of polynomials and entire curves on surfaces', Adv. Math. 225(2) (2010), 1095-1118.

[CZ13] P. Corvaja and U. Zannier, 'Algebraic hyperbolicity of ramified covers of $G_{m}^{2}$ (and integral points on affine subsets of $\mathbb{P}_{2}$ ), J. Differential Geom. 93(3) (2013), 355-377.

[Dem97] J.-P. Demailly, 'Algebraic criteria for Kobayashi hyperbolic projective varieties and jet differentials', in Algebraic geometry - Santa Cruz 1995, Proceedings of Symposia in Pure Mathematics, 62, (American Mathematical Society, Providence, RI, 1997), 285-360.

[Fa194] G. Faltings, 'The general case of S. Lang's conjecture', in Barsotti Symposium in Algebraic Geometry (Abano Terme, 1991), Perspectives in Mathematics, 15 (Academic Press, San Diego, 1994), 175-182.

[FM94] R. Friedman and J. W. Morgan. Smooth Four-Manifolds and Complex Surfaces, Ergebnisse der Mathematik und ihrer Grenzgebiete (3), 27 (Springer-Verlag, Berlin, 1994).

[Gra65] H. Grauert, 'Mordells Vermutung über rationale Punkte auf algebraischen Kurven und Funktionenkörper', Publ. Math. Inst. Hautes Études Sci. 25 (1965), 131-149.

[GW19] J. Guo and J. T.-Y. Wang, 'Asymptotic ged and divisible sequences for entire functions', Trans. Amer. Math. Soc. 371(9) (2019), 6241-6256.

[HR18] S. Hussein and M. Ru, 'A general defect relation and height inequality for divisors in subgeneral position', Asian J. Math. 22(3) (2018), 477-491.

[HS00] M. Hindry and J. H. Silverman, Diophantine Geometry, Graduate Texts in Mathematics, 201 (Springer-Verlag, New York, 2000). An introduction.

[HT00] J. Harris and Y. Tschinkel, 'Rational points on quartics', Duke Math. J. 104(3) (2000), 477-500.

[HT01] B. Hassett and Y. Tschinkel, 'Density of integral points on algebraic varieties', in Rational Points on Algebraic Varieties, Progress in Mathematics, 199, (Birkhäuser, Basel, 2001), 169-197.

[JX20] A. Javanpeykar and J. Xie, 'Finiteness properties of pseudo-hyperbolic varieties', International Mathematics Research Notices, 2020, published online. https://doi.org/10.1093/imrn/rnaa168

[Kaw80] Y. Kawamata, ‘On Bloch's conjecture’, Invent. Math. 57(1) (1980), 97-100.

[Lan86] S. Lang, 'Hyperbolic and Diophantine analysis', Bull. Amer. Math. Soc. (N.S.) 14(2) (1986), 159-205.

[Lan91] S. Lang, Number Theory, III - Diophantine Geometry, Encyclopaedia of Mathematical Sciences, 60 (SpringerVerlag, Berlin, 1991).

[Laz04] R. Lazarsfeld, Positivity in Algebraic Geometry. I, Ergebnisse der Mathematik und ihrer Grenzgebiete (3), 48 (Springer-Verlag, Berlin, 2004).

[Lev09] A. Levin. 'Generalizations of Siegel's and Picard's theorems', Ann. of Math. (2) 170(2) (2009), 609-655.

[Man63] Ju. I. Manin, 'Proof of an analogue of Mordell's conjecture for algebraic curves over function fields', Dokl. Akad. Nauk SSSR 152 (1963), 1061-1063.

[McQ98] M. McQuillan, 'Diophantine approximations and foliations', Publ. Math. Inst. Hautes Études Sci. 87 (1998), 121-174.

[Nog82] J. Noguchi, 'A higher-dimensional analogue of Mordell's conjecture over function fields', Math. Ann. 258(2) (1981/82), 207-212.

[Rou10] E. Rousseau, 'Hyperbolicity of geometric orbifolds', Trans. Amer. Math. Soc. 362(7) (2010), 3799-3826.

[Ru97] M. Ru, 'On a general form of the second main theorem', Trans. Amer. Math. Soc. 349(12) (1997), 5093-5105.

[RV20] M. Ru and P. Vojta, 'A birational Nevanlinna constant and its consequences', Am. J. Math. 142(3) (2020), 957-991.

[Tur17] A. Turchet, 'Fibered threefolds and Lang-Vojta's conjecture over function fields', Trans. Amer. Math. Soc. 369(12) (2017), 8537-8558.

[vBJK19] R. van Bommel, A. Javanpeykar and L. Kamenova, 'Boundedness in families with applications to arithmetic hyperbolicity', Preprint, 2019, arXiv:1907.11225.

[Voj11] P. Vojta, 'Diophantine approximation and Nevanlinna theory', in Arithmetic Geometry, Lecture Notes in Mathematics, 2009 (Springer, Berlin, 2011), 111-224.

[Voj87] P. Vojta, Diophantine Approximations and Value Distribution Theory, Lecture Notes in Mathematics, 1239 (Springer, Berlin, 1987).

[Voj97] P. Vojta, 'On Cartan's theorem and Cartan's conjecture', Amer. J. Math. 119(1) (1997), 1-17.

[Wan04] J. T.-Y. Wang, 'An effective Schmidt's subspace theorem over function fields', Math. Z. 246(4) (2004), 811-844.

[Yam15] K. Yamanoi, 'Holomorphic curves in algebraic varieties of maximal Albanese dimension', Internat. J. Math. 26(6) (2015), 1541006. 